\title{
Motivic Milnor Fibers over Complete Intersection Varieties and their Virtual Betti Numbers
}

\section{Alexander Esterov ${ }^{1}$ and Kiyoshi Takeuchi ${ }^{2}$}

${ }^{1}$ Department of Algebra, Faculty of Mathematics, Complutense

University, Plaza de las Ciencias, 3, Madrid 28040, Spain and ${ }^{2}$ Institute of Mathematics, University of Tsukuba, 1-1-1, Tennodai, Tsukuba, Ibaraki 305-8571, Japan

Correspondence to be sent to: e-mail: takemicro@nifty.com

We study the Jordan normal forms of the local and global monodromies over complete intersection subvarieties of $\mathbb{C}^{n}$ by using the theory of motivic Milnor fibers. The results will be explicitly described by the mixed volumes of the faces of Newton polyhedrons.

\section{Introduction}

In this paper, we study the Jordan normal forms of the local and global monodromies over complete intersection subvarieties of $\mathbb{C}^{n}$ with the help of the theory of motivic Milnor fibers and their Hodge realizations developed by Denef and Loeser [5, 6], Guibert-Loeser-Merle [15] and Matsui-Takeuchi [29] etc. For $2 \leq k \leq n$ let

$$
W=\left\{f_{1}=\cdots=f_{k-1}=0\right\} \supset V=\left\{f_{1}=\cdots=f_{k-1}=f_{k}=0\right\}
$$

be complete intersection subvarieties of $\mathbb{C}^{n}$ such that $0 \in V$. Assume that $W$ and $V$ have isolated singularities at the origin $0 \in \mathbb{C}^{n}$. Then by a fundamental result of Hamm [16], the Milnor fiber $F_{0}$ of $g:=\left.f_{k}\right|_{W}: W \longrightarrow \mathbb{C}$ at the origin 0 has the homotopy type of the 
bouquet of $(n-k)$-spheres. In particular, we have $H^{j}\left(F_{0} ; \mathbb{C}\right) \simeq 0(j \neq 0, n-k)$ and the monodromy operator $\Phi_{n-k, 0}: H^{n-k}\left(F_{0} ; \mathbb{C}\right) \simeq H^{n-k}\left(F_{0} ; \mathbb{C}\right)$ is called the $k$ th principal monodromy of $f:=\left(f_{1}, f_{2}, \ldots, f_{k}\right)$. Under some weak additional conditions, a beautiful formula for the eigenvalues of the $k$ th principal monodromy $\Phi_{n-k, 0}$ was obtained by Oka [33, 34] and Kirillov [20] etc. For related results, see also [11, 26, 27] etc. Moreover, the mixed Hodge structures of the Milnor fiber $F_{0}$ were precisely studied by Ebeling and Steenbrink [9] and Tanabe [45] etc. However, to the best of our knowledge, almost nothing is known for the Jordan normal form of $\Phi_{n-k, 0}$. For a special but important case, see Dimca [7]. In this paper, we propose a method to describe the Jordan normal form of $\Phi_{n-k, 0}$ in terms of the Newton polyhedrons of $f_{1}, f_{2}, \ldots, f_{k}$. Let $\mathcal{M}_{\mathbb{C}}^{\hat{\mu}}$ be the (localized) Grothendieck ring of varieties over $\mathbb{C}$ with (good) action introduced by Denef-Loeser [6]. Then, just as in Denef-Loeser [5, 6], and Guibert et al. [15], we can introduce an element $\mathcal{S}_{g, 0}$ of $\mathcal{M}_{\mathbb{C}}^{\hat{\mu}}$ whose mixed Hodge numbers carry the information of $\Phi_{n-k, 0}$. We call $\mathcal{S}_{g, 0}$ the motivic Milnor fiber of $g: W \longrightarrow \mathbb{C}$ at the origin $0 \in \mathbb{C}^{n}$. We will show that their mixed Hodge numbers can be easily calculated and hence give an algorithm to compute the Jordan normal form of $\Phi_{n-k, 0}$. In order to describe our results more explicitly, assume also that $f_{1}, f_{2}, \ldots, f_{k}$ are convenient and the complete intersection varieties $W$ and $V$ are nondegenerate at the origin $0 \in \mathbb{C}^{n}$ (see Definition 4.6 and [34] etc.). Set

$$
\Gamma_{+}(f):=\Gamma_{+}\left(f_{1}\right)+\Gamma_{+}\left(f_{2}\right)+\cdots+\Gamma_{+}\left(f_{k}\right)
$$

and let $\Gamma_{f}$ be the union of compact faces of $\Gamma_{+}(f)$, where $\Gamma_{+}\left(f_{j}\right) \subset \mathbb{R}_{+}^{n}$ is the Newton polyhedron of $f_{j}$ at the origin $0 \in \mathbb{C}^{n}$. Then for each face $\Theta \prec \Gamma_{+}(f)$ such that $\Theta \subset \Gamma_{f}$, we can naturally define faces $\gamma_{j}^{\Theta}$ of $\Gamma_{+}\left(f_{j}\right)(1 \leq j \leq k)$ such that

$$
\Theta=\gamma_{1}^{\Theta}+\gamma_{2}^{\Theta}+\cdots+\gamma_{k}^{\Theta}
$$
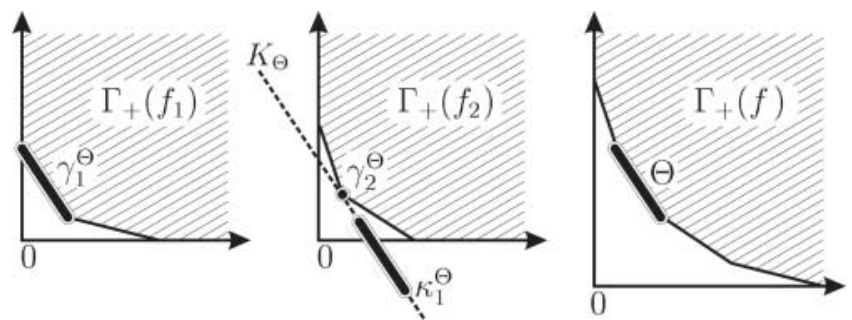

Fig. 1. The case where $k=2$. For $K_{\Theta}$ and $\kappa_{1}^{\Theta}$, see Section 4 . 
By using $\gamma_{j}^{\Theta}$, we define a nondegenerate complete intersection subvariety $Z_{\Delta_{\Theta}}^{*} \subset$ $\left(\mathbb{C}^{*}\right)^{\operatorname{dim} \Theta+1}$ and an element $\left[Z_{\Delta_{\Theta}}^{*}\right] \in \mathcal{M}_{\mathbb{C}}^{\hat{\mu}}$ (see Section 4 for the details). Moreover for such $\Theta \prec \Gamma_{+}(f)$, let $s_{\Theta}$ be the dimension of the minimal coordinate subspace of $\mathbb{R}^{n}$ containing $\Theta$ and set $m_{\Theta}=s_{\Theta}-\operatorname{dim} \Theta-1 \geq 0$. Recall that the element $[\mathbb{L}] \in \mathcal{M}_{\mathbb{C}}^{\hat{\mu}}$ called the Lefschetz motive is defined to be the affine line $\mathbb{L} \simeq \mathbb{C}$ with the trivial action. Following the notations in Denef and Loeser [6, Section 3.1.2 and 3.1.3], we denote by HS $^{\text {mon }}$ the abelian category of Hodge structures with a quasi-unipotent endomorphism. Its Grothendieck group, whose elements are formal differences of those of $\mathrm{HS}^{\mathrm{mon}}$, is denoted by $K_{0}$ (HS ${ }^{\mathrm{mon}}$ ) . Let $\chi_{h}: \mathcal{M}_{\mathbb{C}}^{\hat{\mu}} \longrightarrow \mathrm{K}_{0}\left(\mathrm{HS}^{\mathrm{mon}}\right.$ ) be the Hodge characteristic map in [6] (see also Section 4). For an element $[H] \in \mathrm{K}_{0}\left(\mathrm{HS}^{\mathrm{mon}}\right)$ and $\lambda \in \mathbb{C}^{*}$, we denote by $[H]_{\lambda} \in \mathrm{K}_{0}$ (HS) the $\lambda$-eigenspace of the quasi-unipotent endomorphism on $[H]$.

Theorem 1.1. Assume that $\lambda \in \mathbb{C}^{*} \backslash\{1\}$. Then

(1) In the Grothendieck group $K_{0}(\mathrm{HS})$, we have

$$
\chi_{h}\left(\mathcal{S}_{g, 0}\right)_{\lambda}=\sum_{\Theta \subset \Gamma_{f}, \operatorname{dim} \Theta \geq k-1} \chi_{h}\left((1-\mathbb{L})^{m_{\Theta}} \cdot\left[Z_{\Delta_{\Theta}}^{*}\right)_{\lambda} .\right.
$$

(2) For $i \geq 1$, the number of the Jordan blocks for the eigenvalue $\lambda$ with sizes $\geq i$ in $\Phi_{n-k, 0}: H^{n-k}\left(F_{0} ; \mathbb{C}\right) \simeq H^{n-k}\left(F_{0} ; \mathbb{C}\right)$ is equal to

$$
(-1)^{n-k}\left\{\beta_{n-k-1+i}\left(\mathcal{S}_{g, 0}\right)_{\lambda}+\beta_{n-k+i}\left(\mathcal{S}_{g, 0}\right)_{\lambda}\right\},
$$

where $\beta_{j}\left(\mathcal{S}_{g, 0}\right)_{\lambda}$ is the $j$ th virtual Betti number of $\mathcal{S}_{g, 0}$ (with respect to the eigenvalue $\lambda$ ) defined by the weight $j$-part of the Hodge structure $\chi_{h}\left(\mathcal{S}_{g, 0}\right)$ (see Section 3).

By this theorem, for the determination of the Jordan normal form of $\Phi_{n-k, 0}$ concerning the eigenvalues $\lambda \neq 1$, it suffices to calculate the weight multiplicities of $\chi_{h}\left(\left[Z_{\Delta_{\theta}}^{*}\right]\right) \in \mathrm{K}_{0}\left(\mathrm{HS}^{\mathrm{mon}}\right)$. In Section 4 , by using the Cayley trick in [4, Section 6], we reduce these calculations to those for nondegenerate hypersurfaces in algebraic tori with action. Since we can always calculate the mixed Hodge numbers of such hypersurfaces by our previous results in [29, Section 2], we thus obtain an algorithm to compute the Jordan normal form of $\Phi_{n-k, 0}$. Moreover, in Section 5, we give some closed formulas for the numbers of the Jordan blocks for the eigenvalues $\lambda \neq 1$ with large sizes in $\Phi_{n-k, 0}$. 
The results will be explicitly described by the mixed volumes of the faces $\gamma_{j}^{\Theta}$ of $\Gamma_{+}\left(f_{j}\right)$ $(1 \leq j \leq k)$. See Theorems 5.4 and 5.13 for details. In the course of the proof of these results, Proposition 3.2, which generalizes a result of Khovanskii [19], will play a central role. This proposition expresses an alternating sum of the numbers of certain lattice points defined by a polytope by its volume. We believe that it will be very useful in the study of lattice points in nonintegral polytopes. Indeed, by Proposition 3.2 we can rewrite the main results of [29] much more simply in terms of the volumes of polytopes. Unfortunately, for some technical reasons, we cannot obtain similar results for the eigenvalue 1 (see Remark 4.2 below), using our methods. In Section 6, we will show that our methods are useful also in the study of the monodromies at infinity over complete intersection subvarieties of $\mathbb{C}^{n}$. Even in this global case, we obtain results completely parallel to those in the local case. We thus find a beautiful symmetry between local and global as in [29].

\section{Preliminary Notions and Results}

In this section, we introduce basic notions and results, which will be used in this paper. In this paper, we essentially follow the terminology of $[8,12,17,18]$ etc. For example, for a topological space $X$ we denote by $\mathbf{D}^{b}(X)$ the derived category whose objects are bounded complexes of sheaves of $\mathbb{C}_{X}$-modules on $X$. Moreover if $X$ is an algebraic variety over $\mathbb{C}$, we denote by $\mathbf{D}_{c}^{b}(X)$ the full subcategory of $\mathbf{D}^{b}(X)$ consisting of constructible complexes of sheaves. Let $f: X \longrightarrow \mathbb{C}$ be a nonconstant regular function on an algebraic variety $X$ over $\mathbb{C}$ and set $X_{0}=\{x \in X \mid f(x)=0\} \subset X$. Then we have the nearby cycle functor

$$
\psi_{f}: \mathbf{D}^{b}(X) \longrightarrow \mathbf{D}^{b}\left(X_{0}\right)
$$

which preserves the constructibility (see [8, 18] etc.). As we see in Proposition 2.3, the nearby cycle functor $\psi_{f}$ generalizes the classical notion of Milnor fibers. First, let us recall the definition of Milnor fibers over singular varieties (see for example [44] for a review on this subject). Let $X$ be a subvariety of $\mathbb{C}^{m}$ and $f: X \longrightarrow \mathbb{C}$ a nonconstant regular function on $X$. Namely we assume that there exists a polynomial function $\tilde{f}: \mathbb{C}^{m} \longrightarrow \mathbb{C}$ on $\mathbb{C}^{m}$ such that $\left.\tilde{f}\right|_{X}=f$. For simplicity, assume also that the origin $0 \in \mathbb{C}^{m}$ is contained in $X_{0}=\{x \in X \mid f(x)=0\}$. Then the following lemma is well known. 
Lemma 2.1 ([23, Theorem 1.1]). For sufficiently small $\varepsilon>0$, there exists $\eta_{0}>0$ with $0<\eta_{0} \ll \varepsilon$ such that for $0<\forall \eta<\eta_{0}$ the restriction of $f$ :

$$
X \cap B(0 ; \varepsilon) \cap \tilde{f}^{-1}(D(0 ; \eta) \backslash\{0\}) \longrightarrow D(0 ; \eta) \backslash\{0\}
$$

is a topological fiber bundle over the punctured disk $D(0 ; \eta) \backslash\{0\}:=\{z \in \mathbb{C}|0<| z \mid<\eta\}$, where $B(0 ; \varepsilon)$ is the open ball in $\mathbb{C}^{m}$ with radius $\varepsilon$ centered at the origin.

Definition 2.2. A fiber of the above fibration is called the Milnor fiber of the function $f: X \longrightarrow \mathbb{C}$ at $0 \in X_{0}$ and we denote it by $F_{0}$.

Proposition 2.3 ([8, Proposition 4.2.2]). There exists a natural isomorphism

$$
H^{j}\left(F_{0} ; \mathbb{C}\right) \simeq H^{j}\left(\psi_{f}\left(\mathbb{C}_{X}\right)\right)_{0}
$$

for any $j \in \mathbb{Z}$.

Recall also that in the above situation, as in the case of polynomial functions over $\mathbb{C}^{n}$ (see Milnor [31]), we can define the Milnor monodromy operators

$$
\Phi_{j, 0}: H^{j}\left(F_{0} ; \mathbb{C}\right) \stackrel{\sim}{\longrightarrow} H^{j}\left(F_{0} ; \mathbb{C}\right) \quad(j=0,1, \ldots) .
$$

For the classical case where $X=\mathbb{C}^{m}$ see $[1,3,21,22,29,31,34,45,47]$ etc. Similarly, also for any $y \in X_{0}=\{x \in X \mid f(x)=0\}$ we can define the Milnor fiber $F_{Y}$ and its monodromies $\Phi_{j, y}$. The notion of Milnor monodromies can be also generalized as follows. Let $\mathcal{F} \in$ $\mathbf{D}_{C}^{b}(X)$. Then there exists a monodromy automorphism

$$
\Phi(\mathcal{F}): \psi_{f}(\mathcal{F}) \stackrel{\sim}{\longrightarrow} \psi_{f}(\mathcal{F})
$$

of $\psi_{f}(\mathcal{F})$ in $\mathbf{D}_{c}^{b}\left(X_{0}\right)$ (see $[8,18]$ etc.).

Next we recall Bernstein-Khovanskii-Kushnirenko's theorem [19].

Definition 2.4. Let $g(x)=\sum_{v \in \mathbb{Z}^{n}} a_{v} x^{v}$ be a Laurent polynomial on $\left(\mathbb{C}^{*}\right)^{n}\left(a_{v} \in \mathbb{C}\right)$.

(1) We call the convex hull of $\operatorname{supp}(g):=\left\{v \in \mathbb{Z}^{n} \mid a_{v} \neq 0\right\} \subset \mathbb{Z}^{n} \subset \mathbb{R}^{n}$ in $\mathbb{R}^{n}$ the Newton polytope of $g$ and denote it by $\mathrm{NP}(g)$. 
(2) For a vector $u \in \mathbb{R}^{n}$, we set

$$
\Gamma(g ; u):=\left\{v \in \operatorname{NP}(g) \mid\langle u, v\rangle=\min _{w \in \mathrm{NP}(g)}\langle u, w\rangle\right\}
$$

where for $u=\left(u_{1}, \ldots, u_{n}\right)$ and $v=\left(v_{1}, \ldots, v_{n}\right)$ we set $\langle u, v\rangle=\sum_{i=1}^{n} u_{i} v_{i}$. We call $\Gamma(g ; u)$ the supporting face of $u$ in $\operatorname{NP}(g)$.

(3) For a vector $u \in \mathbb{R}^{n}$, we define the $u$-part of $g$ by

$$
g^{u}(x):=\sum_{v \in \Gamma(g ; u) \cap \mathbb{Z}^{n}} a_{v} x^{v}
$$

Definition 2.5. Let $g_{1}, g_{2}, \ldots, g_{p}$ be Laurent polynomials on $\left(\mathbb{C}^{*}\right)^{n}$. Then we say that the subvariety $Z^{*}=\left\{x \in\left(\mathbb{C}^{*}\right)^{n} \mid g_{1}(x)=g_{2}(x)=\cdots=g_{p}(x)=0\right\}$ of $\left(\mathbb{C}^{*}\right)^{n}$ is a nondegenerate complete intersection if for any vector $u \in \mathbb{R}^{n}$ the $p$-form $d g_{1}^{u} \wedge d g_{2}^{u} \wedge \cdots \wedge d g_{p}^{u}$ does not vanish on $\left\{x \in\left(\mathbb{C}^{*}\right)^{n} \mid g_{1}^{u}(x)=\cdots=g_{p}^{u}(x)=0\right\}$.

Theorem 2.6 (Bernstein-Khovanskii-Kushnirenko's theorem, see [19] etc.). Let $g_{1}, g_{2}, \ldots, g_{p}$ be Laurent polynomials on $\left(\mathbb{C}^{*}\right)^{n}$. Assume that the subvariety $Z^{*}=\left\{x \in\left(\mathbb{C}^{*}\right)^{n} \mid g_{1}(x)=g_{2}(x)=\cdots=g_{p}(x)=0\right\}$ of $\left(\mathbb{C}^{*}\right)^{n}$ is a nondegenerate complete intersection. Set $\Delta_{i}:=\operatorname{NP}\left(g_{i}\right)$ for $i=1, \ldots, p$. Then the Euler characteristic $\chi\left(Z^{*}\right)$ of $Z^{*}$ is given by

$$
\chi\left(Z^{*}\right)=(-1)^{n-p} \sum_{\begin{array}{c}
a_{1}, \ldots, a_{p} \geq 1 \\
a_{1}+\cdots+a_{p}=n
\end{array}} \operatorname{MV}(\underbrace{\Delta_{1}, \ldots, \Delta_{1}}_{a_{1} \text {-times }}, \ldots, \underbrace{\Delta_{p}, \ldots, \Delta_{p}}_{a_{p} \text {-times }})
$$

where $\operatorname{MV}(\underbrace{\Delta_{1}, \ldots, \Delta_{1}}_{a_{1} \text {-times }}, \ldots, \underbrace{\Delta_{p}, \ldots, \Delta_{p}}_{a_{p} \text {-times }}) \in \mathbb{Z}$ is the normalized $n$-dimensional mixed volume with respect to the lattice $\mathbb{Z}^{n} \subset \mathbb{R}^{n}$.

Remark 2.7. Let $Q_{1}, Q_{2}, \ldots, Q_{n}$ be polytopes in $\mathbb{R}^{n}$. Then their normalized $n$-dimensional mixed volume $\operatorname{MV}\left(Q_{1}, Q_{2}, \ldots, Q_{n}\right) \in \mathbb{R}_{+}$is given by the formula

$$
\operatorname{MV}\left(Q_{1}, Q_{2}, \ldots, Q_{n}\right)=\frac{1}{n !} \sum_{k=1}^{n}(-1)^{n-k}\left\{\sum_{\substack{I \subset\{1, \ldots, n\} \\ \sharp I=k}} \operatorname{Vol}_{\mathbb{Z}}\left(\sum_{i \in I} O_{i}\right)\right\},
$$

where $\operatorname{Vol}_{\mathbb{Z}}(*) \in \mathbb{Z}$ is the normalized $n$-dimensional volume (i.e., the $n$ ! times the usual volume). Note that if $Q_{1}, \ldots, Q_{n}$ are integral polytopes $\operatorname{MV}\left(Q_{1}, Q_{2}, \ldots, Q_{n}\right) \in \mathbb{Z}_{+}$. 
Finally we shall introduce our recent results in [29, Section 2]. From now on, let us fix an element $\tau=\left(\tau_{1}, \ldots, \tau_{n}\right) \in T:=\left(\mathbb{C}^{*}\right)^{n}$ and let $g$ be a Laurent polynomial on $\left(\mathbb{C}^{*}\right)^{n}$ such that $Z^{*}=\left\{x \in\left(\mathbb{C}^{*}\right)^{n} \mid g(x)=0\right\}$ is nondegenerate and invariant by the automorphism $l_{\tau}:\left(\mathbb{C}^{*}\right)^{n} \underset{\tau \times}{\stackrel{\sim}{\longrightarrow}}\left(\mathbb{C}^{*}\right)^{n}$ induced by the multiplication by $\tau$. Set $\Delta=\operatorname{NP}(g)$ and for simplicity assume that $\operatorname{dim} \Delta=n$. Then there exists $\beta \in \mathbb{C}$ such that $l_{\tau}^{*} g=g \circ l_{\tau}=\beta g$. This implies that for any vertex $v$ of $\Delta=\mathrm{NP}(g)$, we have $\tau^{v}=\tau_{1}^{v_{1}} \cdots \tau_{n}^{v_{n}}=\beta$. Moreover by the condition $\operatorname{dim} \Delta=n$ we see that $\tau_{1}, \tau_{2}, \ldots, \tau_{n}$ are roots of unity. For $p, q \geq 0$ and $k \geq 0$, let $h^{p, q}\left(H_{c}^{k}\left(Z^{*} ; \mathbb{C}\right)\right)$ be the mixed Hodge number of $H_{c}^{k}\left(Z^{*} ; \mathbb{C}\right)$ and set

$$
e^{p, q}\left(Z^{*}\right)=\sum_{k}(-1)^{k} h^{p, q}\left(H_{c}^{k}\left(Z^{*} ; \mathbb{C}\right)\right)
$$

as in [4]. The above automorphism of $\left(\mathbb{C}^{*}\right)^{n}$ induces a morphism of mixed Hodge structures $l_{\tau}^{*}: H_{c}^{k}\left(Z^{*} ; \mathbb{C}\right) \stackrel{\sim}{\longrightarrow} H_{c}^{k}\left(Z^{*} ; \mathbb{C}\right)$ and hence $\mathbb{C}$-linear transformations on the $(p, q)$-parts $H_{c}^{k}\left(Z^{*} ; \mathbb{C}\right)^{p, q}$ of $H_{c}^{k}\left(Z^{*} ; \mathbb{C}\right)$. For $\alpha \in \mathbb{C}$, let $h^{p, q}\left(H_{c}^{k}\left(Z^{*} ; \mathbb{C}\right)\right)_{\alpha}$ be the dimension of the $\alpha$ eigenspace $H_{c}^{k}\left(Z^{*} ; \mathbb{C}\right)_{\alpha}^{p, q}$ of this automorphism of $H_{C}^{k}\left(Z^{*} ; \mathbb{C}\right)^{p, q}$ and set

$$
e^{p, q}\left(Z^{*}\right)_{\alpha}=\sum_{k}(-1)^{k} h^{p, q}\left(H_{c}^{k}\left(Z^{*} ; \mathbb{C}\right)\right)_{\alpha}
$$

Since we have $l_{\tau}^{r}=\mathrm{id}_{Z^{*}}$ for $r \gg 0$, these numbers are zero unless $\alpha$ is a root of unity. Moreover we have

$$
e^{p, q}\left(Z^{*}\right)=\sum_{\alpha \in \mathbb{C}} e^{p, q}\left(Z^{*}\right)_{\alpha}, \quad e^{p, q}\left(Z^{*}\right)_{\alpha}=e^{q, p}\left(Z^{*}\right)_{\bar{\alpha}}
$$

In this situation, along the lines of Danilov and Khovanskii [4], we can give an algorithm for computing these numbers $e^{p, q}\left(Z^{*}\right)_{\alpha}$ as follows. First of all, as in [4, Section 3], we obtain the following Lefschetz type theorem.

Proposition 2.8 ([29, Proposition 2.6]). For $p, q \geq 0$ such that $p+q>n-1$, we have

$$
e^{p, q}\left(Z^{*}\right)_{\alpha}= \begin{cases}(-1)^{n+p+1}\left(\begin{array}{c}
n \\
p+1
\end{array}\right) & (\alpha=1 \text { and } p=q) \\
0 & \text { (otherwise) }\end{cases}
$$


8 A. Esterov and K. Takeuchi

For a vertex $w$ of $\Delta$, consider the translated polytope $\Delta^{w}:=\Delta-w$ such that $0 \prec \Delta^{w}$ and $\tau^{v}=1$ for any vertex $v$ of $\Delta^{w}$. Then for $\alpha \in \mathbb{C}$ and $k \geq 0$ set

$$
l^{*}(k \Delta)_{\alpha}=\sharp\left\{v \in \operatorname{Int}\left(k \Delta^{w}\right) \cap \mathbb{Z}^{n} \mid \tau^{v}=\alpha\right\} \in \mathbb{Z}_{+}:=\mathbb{Z}_{\geq 0 .}
$$

For the special case $\alpha=1$ see Macdonald [25] etc. We can easily see that these numbers $l^{*}(k \Delta)_{\alpha}$ do not depend on the choice of the vertex $w$ of $\Delta$. Note that for $k=0$ we have $l^{*}(k \Delta)_{\alpha}=0$. Next, define a formal power series $P_{\alpha}(\Delta ; t)=\sum_{i \geq 0} \varphi_{\alpha, i}(\Delta) t^{i}$ by

$$
P_{\alpha}(\Delta ; t)=(1-t)^{n+1}\left\{\sum_{k \geq 0} l^{*}(k \Delta)_{\alpha} t^{k}\right\} .
$$

Then obviously we have $\varphi_{\alpha, 0}(\Delta)=0$. Moreover, we can easily show that $P_{\alpha}(\Delta ; t)$ is actually a polynomial as in [4, Section 4.4].

Theorem 2.9 ([29, Theorem 2.7]). In the situation above, we have

$$
\sum_{q} e^{p, q}\left(Z^{*}\right)_{\alpha}= \begin{cases}(-1)^{p+n+1}\left(\begin{array}{c}
n \\
p+1
\end{array}\right)+(-1)^{n+1} \varphi_{\alpha, n-p}(\Delta) & (\alpha=1), \\
(-1)^{n+1} \varphi_{\alpha, n-p}(\Delta) & (\alpha \neq 1) .\end{cases}
$$

(we used the convention $\left(\begin{array}{l}a \\ b\end{array}\right)=0(0 \leq a<b)$ for binomial coefficients).

By Proposition 2.8 and Theorem 2.9, we obtain an algorithm to calculate the numbers $e^{p, q}\left(Z^{*}\right)_{\alpha}$ of the nondegenerate hypersurface $Z^{*} \subset\left(\mathbb{C}^{*}\right)^{n}$ for any $\alpha \in \mathbb{C}$ as in [4, Section 5.2]. Indeed for a projective toric compactification $X$ of $\left(\mathbb{C}^{*}\right)^{n}$ such that the closure $\bar{Z}^{*}$ of $Z^{*}$ in $X$ is smooth (see $[13,32]$ etc.), the variety $\bar{Z}^{*}$ is smooth projective and hence there exists a perfect pairing

$$
H^{p, q}\left(\bar{Z}^{*} ; \mathbb{C}\right)_{\alpha} \times H^{n-1-p, n-1-q}\left(\bar{Z}^{*} ; \mathbb{C}\right)_{\alpha^{-1}} \longrightarrow \mathbb{C}
$$

for any $p, q \geq 0$ and $\alpha \in \mathbb{C}^{*}$ (see, e.g., [49, Section 5.3.2]). Therefore, we obtain equalities $e^{p, q}\left(\bar{Z}^{*}\right)_{\alpha}=e^{n-1-p, n-1-q}\left(\bar{Z}^{*}\right)_{\alpha^{-1}}$, which are necessary to proceed the algorithm in [4, Section 5.2]. The following notion is very useful to construct such compactifications of $\left(\mathbb{C}^{*}\right)^{n}$. 
Definition 2.10 ([4]).

(1) Let $\Delta$ be an $n$-dimensional integral polytope in $\left(\mathbb{R}^{n}, \mathbb{Z}^{n}\right)$. For a vertex $w$ of $\Delta$, we define a closed convex cone $\operatorname{Con}(\Delta, w)$ by $\operatorname{Con}(\Delta, w)=\{r \cdot(v-w) \mid r \in$ $\left.\mathbb{R}_{+}, v \in \Delta\right\} \subset \mathbb{R}^{n}$.

(2) Let $\Delta$ and $\Delta^{\prime}$ be two $n$-dimensional integral polytopes in $\left(\mathbb{R}^{n}, \mathbb{Z}^{n}\right)$. We denote by $\operatorname{som}(\Delta)\left(\right.$ resp. $\left.\operatorname{som}\left(\Delta^{\prime}\right)\right)$ the set of vertices of $\Delta\left(\right.$ resp. $\left.\Delta^{\prime}\right)$. Then we say that $\Delta^{\prime}$ majorizes $\Delta$ if there exists a map $\Psi: \operatorname{som}\left(\Delta^{\prime}\right) \longrightarrow \operatorname{som}(\Delta) \operatorname{such}$ that $\operatorname{Con}(\Delta, \Psi(w)) \subset \operatorname{Con}\left(\Delta^{\prime}, w\right)$ for any $w \in \operatorname{som}\left(\Delta^{\prime}\right)$.

Note that if $\Delta^{\prime}$ majorizes $\Delta$ the map $\Psi: \operatorname{som}\left(\Delta^{\prime}\right) \longrightarrow \operatorname{som}(\Delta)$ is unique (see [4]). For an $n$-dimensional integral polytope $\Delta$ in $\left(\mathbb{R}^{n}, \mathbb{Z}^{n}\right)$, we denote by $X_{\Delta}$ the (projective) toric variety associated with the dual fan of $\Delta$. Recall also that if $\Delta^{\prime}$ majorizes $\Delta$ then there exists a natural morphism $X_{\Delta^{\prime}} \longrightarrow X_{\Delta}$. Now we return to the original situation. For $\alpha \in \mathbb{C}$ we define the $\alpha$-Euler characteristic $\chi\left(Z^{*}\right)_{\alpha} \in \mathbb{Z}$ of $Z^{*} \subset\left(\mathbb{C}^{*}\right)^{n}$ by

$$
\chi\left(Z^{*}\right)_{\alpha}=\sum_{p, q} e^{p, q}\left(Z^{*}\right)_{\alpha} .
$$

Then we have the following result.

Proposition 2.11. For any $\alpha \in \mathbb{C}$, we have

$$
\chi\left(Z^{*}\right)_{\alpha}= \begin{cases}(-1)^{n-1}+\sum_{k=1}^{n}(-1)^{k+1}\left(\begin{array}{l}
n \\
k
\end{array}\right) l^{*}(k \Delta)_{\alpha} & (\alpha=1), \\
\sum_{k=1}^{n}(-1)^{k+1}\left(\begin{array}{l}
n \\
k
\end{array}\right) l^{*}(k \Delta)_{\alpha} & (\alpha \neq 1) .\end{cases}
$$

Proof. By Theorem 2.9 we have

$$
\chi\left(Z^{*}\right)_{\alpha}= \begin{cases}(-1)^{n+1}\left\{1+\varphi_{1,1}(\Delta)+\cdots+\varphi_{1, n}(\Delta)\right\} & (\alpha=1), \\ (-1)^{n+1}\left\{\varphi_{\alpha, 1}(\Delta)+\cdots+\varphi_{\alpha, n}(\Delta)\right\} & (\alpha \neq 1) .\end{cases}
$$


Then the result follows from

$$
\begin{aligned}
\sum_{j=1}^{n} \varphi_{\alpha, j}(\Delta) & =\sum_{j=1}^{n} \sum_{i=0}^{j}(-1)^{i}\left(\begin{array}{c}
n+1 \\
i
\end{array}\right) l^{*}\left((j-i)^{\prime} \Delta\right)_{\alpha} \\
& =\sum_{k=1}^{n}(-1)^{n-k}\left(\begin{array}{l}
n \\
k
\end{array}\right) l^{*}(k \Delta)_{\alpha} .
\end{aligned}
$$

From now on, assume also that for any vertex $v$ of $\Delta$ we have $\tau^{v}=1$. Let $L_{\tau} \subset \mathbb{Z}^{n}$ be the sublattice of $\mathbb{Z}^{n}$ defined by $L_{\tau}=\left\{v \in \mathbb{Z}^{n} \mid \tau^{v}=1\right\}$. For an integral polytope $\square$ in $\mathbb{R}^{n}$, we set $\downarrow(\square)=(-1)^{\operatorname{dim} \square_{\sharp}}$ rel.int $\left.(\square) \cap L_{\tau}\right\}$, where rel.int $(\square)$ is the relative interior of $\square$. Note that if $\operatorname{dim} \square=0$ then we have $\boxminus(\square)=1$ or 0 depending on whether $\square$ is a point in $L_{\tau}$ or not. Then for any $\alpha \in \mathbb{C}$ such that $\left\{v \in \mathbb{Z}^{n} \mid \tau^{v}=\alpha\right\} \neq \emptyset$, by taking a point $w(\alpha) \in\left\{v \in \mathbb{Z}^{n} \mid \tau^{v}=\right.$ $\alpha$, Proposition 2.11 can be rewritten as follows.

Proposition 2.12. For any $\alpha \in \mathbb{C}$ we have

$$
(-1)^{n-1} \chi\left(Z^{*}\right)_{\alpha}=\sum_{k=0}^{n}(-1)^{k}\left(\begin{array}{l}
n \\
k
\end{array}\right) \cdot \natural(k \Delta-w(\alpha)) .
$$

More generally, for any subvariety $Y^{*} \subset\left(\mathbb{C}^{*}\right)^{n}$ that is invariant by $l_{\tau}:\left(\mathbb{C}^{*}\right)^{n} \stackrel{\sim}{\longrightarrow}$ $\left(\mathbb{C}^{*}\right)^{n}, p, q \geq 0$ and $\alpha \in \mathbb{C}$ we can define $e^{p, q}\left(Y^{*}\right)_{\alpha} \in \mathbb{Z}$ satisfying similar properties. For example, fix integral polytopes $\Delta_{1}, \ldots, \Delta_{k}$ in $\mathbb{R}^{n}$ whose all vertices lie in $L_{\tau}$ and set $N=\sum_{j=1}^{k} \sharp\left(\Delta_{j} \cap L_{\tau}\right)$. Let $S \simeq \mathbb{C}^{N}$ be the set of $k$-tuples $\left(g_{1}, \ldots, g_{k}\right)$ of Laurent polynomials on $\left(\mathbb{C}^{*}\right)^{n}$ such that suppg $g_{j} \subset \Delta_{j} \cap L_{\tau}$. Here we regard $S$ as the affine space $\mathbb{C}^{N}$ consisting of the coefficients of $\left(g_{1}, \ldots, g_{k}\right)$. Let $S_{\text {gen }}$ be the subset of $S$ consisting of $k$-tuples $\left(g_{1}, \ldots, g_{k}\right)$ such that $\mathrm{NP}\left(g_{j}\right)=\Delta_{j}$ and $Z\left(g_{1}, \ldots, g_{k}\right)^{*}:=\left\{x \in\left(\mathbb{C}^{*}\right)^{n} \mid g_{1}(x)=\cdots=g_{k}(x)=0\right\}$ is a nondegenerate complete intersection (C.I.). Then $S_{\text {gen }}$ is open dense in $S \simeq \mathbb{C}^{N}$ and for any $\left(g_{1}, \ldots, g_{k}\right) \in S_{\text {gen }}$ the C.I. subvariety $Z\left(g_{1}, \ldots, g_{k}\right)^{*} \subset\left(\mathbb{C}^{*}\right)^{n}$ is invariant by $l_{\tau}$. Hence the numbers $e^{p, q}\left(Z\left(g_{1}, \ldots, g_{k}\right)^{*}\right)_{\alpha} \in \mathbb{Z}$ are defined.

Lemma 2.13. For any $p, q \geq 0$ and $\alpha \in \mathbb{C}$ the integer $e^{p, q}\left(Z\left(g_{1}, \ldots, g_{k}\right)^{*}\right)_{\alpha}$ does not depend on $\left(g_{1}, \ldots, g_{k}\right) \in S_{\text {gen }}$. 
Proof. Set $m=\operatorname{dim}\left(\Delta_{1}+\cdots+\Delta_{k}\right)$. Then for any $\left(g_{1}, \ldots, g_{k}\right) \in S_{\text {gen }}$ we have $Z\left(g_{1}, \ldots, g_{k}\right)^{*} \simeq\left(\mathbb{C}^{*}\right)^{n-m} \times Z^{\prime}\left(g_{1}, \ldots, g_{k}\right)^{*}$ for a nondegenerate complete intersection $Z^{\prime}\left(g_{1}, \ldots, g_{k}\right)^{*} \subset\left(\mathbb{C}^{*}\right)^{m}$ and $l_{\tau}: Z\left(g_{1}, \ldots, g_{k}\right)^{*} \stackrel{\sim}{\longrightarrow} Z\left(g_{1}, \ldots, g_{k}\right)^{*}$ is homotopic to $\operatorname{id}_{\left(\mathbb{C}^{*}\right)^{n-m}} \times l_{\tau^{\prime}}$ for some $\tau^{\prime} \in\left(\mathbb{C}^{*}\right)^{m}$. So we may assume that $\operatorname{dim}\left(\Delta_{1}+\cdots+\Delta_{k}\right)=n$ from the first. Let $\Delta$ be an integral polytope in $\mathbb{R}^{n}$ that majorizes $\Delta_{1}+\cdots+\Delta_{k}$. Then by subdividing the dual fan $\Sigma_{1}$ of $\Delta$ in $\mathbb{R}^{n}$, we obtain a complete fan $\Sigma$ such that the toric variety $X_{\Sigma}$ associated to it is a smooth compactification of $\left(\mathbb{C}^{*}\right)^{n}$. By construction, the closure $\overline{Z\left(g_{1}, \ldots, g_{k}\right)^{*}}$ of $Z\left(g_{1}, \ldots, g_{k}\right)^{*}$ in $X_{\Sigma}$ is smooth for any $\left(g_{1}, \ldots, g_{k}\right) \in S_{\text {gen, }}$ and hence we obtain a family $\pi: \bar{Z}^{*} \longrightarrow S_{\text {gen }}$ of smooth projective varieties over $S_{\text {gen }}$. By using the relative de Rham complex $\Omega_{\bar{Z}^{*} / S_{\text {gen }}}$ of $\pi: \bar{Z}^{*} \longrightarrow S_{\text {gen }}$, for each $i \geq 0$ we obtain a holomorphic variation $\mathcal{H}^{i}=R^{i} \pi_{*}\left(\Omega_{\bar{Z}^{*} / S_{\text {gen }}}^{\cdot}\right.$ ) of (pure) Hodge structures on $S_{\text {gen }}$. Its Hodge filtration $F^{p} \mathcal{H}^{i} \subset \mathcal{H}^{i}$ $(p \geq 0)$ is defined by $F^{p} \mathcal{H}^{i}=R^{i} \pi_{*}\left(\Omega_{\bar{Z}^{*} / S_{\text {gen }}}^{\geqslant p}\right)$ (see [49, Section 10.2.1] etc.). For $p \geq 0$ let

$$
\Phi(p): F^{p} \mathcal{H}^{i} \longrightarrow F^{p} \mathcal{H}^{i}
$$

be the $\mathcal{O}_{S_{\text {gen }}}$-linear endomorphism of the locally free $\mathcal{O}_{S_{\text {gen }}}$-module $F^{p} \mathcal{H}^{i}$ induced by the pull-back of $\Omega_{\bar{Z}^{*} / S_{\text {gen }}}^{\geqslant p}$ by $l_{\tau} \times \operatorname{id}_{S_{\text {gen }}}$. Then by $(\Phi(p))^{N}=\mathrm{id}(N \gg 0)$ we obtain a decomposition

$$
F^{p} \mathcal{H}^{i}=\bigoplus_{\alpha \in \mathbb{C}^{*}}\left(F^{p} \mathcal{H}^{i}\right)_{\alpha}
$$

of $F^{p} \mathcal{H}^{i}$ into the eigenspaces of $\Phi(p)$. Hence $\left(F^{p} \mathcal{H}^{i}\right)_{\alpha}$ are also locally free over $\mathcal{O}_{S_{\text {gen }}}$. Then by [49, Theorem 10.10], the functions $e^{p, q}\left(\overline{Z\left(g_{1}, \ldots, g_{k}\right)^{*}}\right)_{\alpha}$ on $S_{\text {gen }}$ are constant. Moreover we can easily prove a similar statement also for $e^{p, q}\left(Z\left(g_{1}, \ldots, g_{k}\right)^{*}\right)_{\alpha}$ by induction on $n$.

\section{Combinatorial Results and their Applications}

In this section, we shall describe the $\alpha$-Euler characteristic $\chi\left(Z^{*}\right)_{\alpha} \in \mathbb{Z}$ of the nondegenerate hypersurface $Z^{*} \subset\left(\mathbb{C}^{*}\right)^{n}$ introduced in Section 2 in terms of the volume of its Newton polytope $\Delta$. For this purpose, we first consider the following more general situation. Let $L \subset \mathbb{Z}^{n}$ be a sublattice of rank $n$. For a bounded subset $A$ of $\mathbb{R}^{n}$ (resp. a polytope $\square$ in $\mathbb{R}^{n}$ ) we set $\sharp A=\sharp(A \cap L) \in \mathbb{Z}_{+}$(resp. $\square(\square)=(-1)^{\operatorname{dim} \square} \sharp\{\operatorname{rel}$.int $(\square) \cap L\} \in \mathbb{Z}$ ) for short. Let $\Delta_{1}, \Delta_{2}, \ldots, \Delta_{n}$ be integral polytopes in $\mathbb{R}^{n}$ whose all vertices lie in $L$. For a subset $J \subset\{1,2, \ldots, n\}$, we set $\Delta_{J}=\sum_{j \in J} \Delta_{j}$. In particular, for $J=\emptyset$ we set $\Delta_{J}=\{0\}$. Then the following result is well known. 
Theorem 3.1 (Khovanskii [19]). In the situation above, we have

$$
\sum_{J \subset\{1,2, \ldots, n\}}(-1)^{\sharp J} \bigsqcup\left(\Delta_{J}\right)=\operatorname{MV}\left(\Delta_{1}, \Delta_{2}, \ldots, \Delta_{n}\right),
$$

where $\operatorname{MV}\left(\Delta_{1}, \ldots, \Delta_{n}\right) \in \mathbb{Z}_{+}$is the normalized $n$-dimensional mixed volume of $\Delta_{1}, \Delta_{2}, \ldots, \Delta_{n}$ with respect to the lattice $L$.

From now on, we will generalize this theorem as follows. Let $\Delta_{1}, \ldots, \Delta_{n}$ be as above and pick another polytope $\Delta_{0}$ in $\mathbb{R}^{n}$ (which is not assumed to be integral). Also for a subset $I \subset\{0,1,2, \ldots, n\}$, we set $\Delta_{I}=\sum_{j \in I} \Delta_{j}$.

Proposition 3.2. In the situation above, we have

$$
\begin{aligned}
\operatorname{MV}\left(\Delta_{1}, \Delta_{2}, \ldots, \Delta_{n}\right) & =\sum_{J \subset\{1,2, \ldots, n\}}(-1)^{\sharp J} \sharp\left(\Delta_{\{0\} \sqcup J}\right) \\
& =\sum_{J \subset\{1,2, \ldots, n\}}(-1)^{n-\sharp J} \sharp\left(\Delta_{\{0\} \sqcup J}\right) .
\end{aligned}
$$

Proof. The proof proceeds in three steps.

(A) Assume that there exists $1 \leq j \leq n$ such that $\operatorname{dim} \Delta_{j}=0$. In this case, the mixed volume $\operatorname{MV}\left(\Delta_{1}, \ldots, \Delta_{n}\right)$ is zero and the other two terms in (32) also vanish, because for each $J \subset\{1,2, \ldots, n\}$ such that $j \notin J$ we have the cancelling

$$
(-1)^{\sharp(J \sqcup\{j\})} \bigsqcup\left(\Delta_{\{0\} \sqcup J \sqcup\{j\}}\right)+(-1)^{\sharp J} \sqcup\left(\Delta_{\{0\} \sqcup J}\right)=0,
$$

etc.

(B) Assume that $\Delta_{1}, \Delta_{2}, \ldots, \Delta_{n}$ are linearly independent segments and $\Delta_{0}$ consists of one point $p \in \mathbb{R}^{n}$. In this case, for each $1 \leq j \leq n$ by taking a vertex $q_{j}$ of the segment $\Delta_{j}$, we set $\hat{\Delta}_{j}=\Delta_{j} \backslash\left\{q_{j}\right\}$. Then we have

$$
\begin{aligned}
\sum_{J \subset\{1,2, \ldots, n\}}(-1)^{\sharp J} \downarrow\left(\Delta_{\{0\} \sqcup J}\right) & =\sum_{J \subset\{1,2, \ldots, n\}}(-1)^{n-\sharp J} \sharp\left(\Delta_{\{0\} \sqcup J}\right) \\
& =\sharp\left(\hat{\Delta}_{1}+\cdots+\hat{\Delta}_{n}+p\right) .
\end{aligned}
$$

Moreover we can easily see that the last term is equal to $\operatorname{MV}\left(\Delta_{1}, \ldots, \Delta_{n}\right)$. 
(C) Now we consider the general case. For a polytope $\square$ in $\mathbb{R}^{n}$ let $\mathbf{1}_{\square}: \mathbb{R}^{n} \longrightarrow$ $\{0,1\}$ (resp. $\rho_{\square}: \mathbb{R}^{n} \longrightarrow\{0, \pm 1\}$ ) be the characteristic function of $\square$ (resp. the function defined by $\left.\rho_{\square}=(-1)^{\operatorname{dim} \square} \mathbf{1}_{\text {rel.int( }(\square)}\right)$. In particular, for any point $p \in$ $\mathbb{R}^{n}$ we have $\rho_{\{p\}}=\mathbf{1}_{\{p\}}$. If $\square$ and $\square^{\prime}$ are polytopes in $\mathbb{R}^{n}$ and $\square$ majorizes $\square^{\prime}$, then for a face $\Gamma \prec \square$ of $\square$ we denote by $\Gamma^{\prime}$ the corresponding face of $\square^{\prime}$. The proof of the following lemma is very easy and left to the reader.

Lemma 3.3. In the situation above, we have

$$
\sum_{\Gamma<\square}(-1)^{\operatorname{dim} \Gamma} \rho_{\Gamma^{\prime}}=\mathbf{1}_{\square}, \quad \sum_{\Gamma<\square}(-1)^{\operatorname{dim} \Gamma} \mathbf{1}_{\Gamma^{\prime}}=\rho_{\square^{\prime}} .
$$

Actually, we need this lemma in the following special setting.

Lemma 3.4. Let $\square$ and $\square^{\prime}$ be as above and $l \subset \mathbb{R}^{n}$ a closed ray (i.e., a closed half segment $\simeq[0, \infty)$ ) in $\mathbb{R}^{n}$ whose extremal point is the origin $0 \in \mathbb{R}^{n}$. Then we have

$$
\sum_{\Gamma}(-1)^{\operatorname{dim} \Gamma+1} \rho_{\Gamma^{\prime}+l}=\mathbf{1}_{\square^{\prime}+l}, \quad \sum_{\Gamma}(-1)^{\operatorname{dim} \Gamma+1} \mathbf{1}_{\Gamma^{\prime}+l}=\rho_{\square^{\prime}+l},
$$

where $\Gamma$ ranges through the bounded faces of $\square+l$ (they are also faces of $\square$ ) and $\Gamma^{\prime}$ is the face of $\square^{\prime}$ that corresponds to $\Gamma \prec \square$.

Now we return to the proof of Proposition 3.2. Let $f_{0}, f_{1}, \ldots, f_{n}: \mathbb{R}^{n} \longrightarrow \mathbb{R}$ be polynomials of order $\leq 1$ such that $\left.f_{j}\right|_{\Delta_{j}}>0$. For $0 \leq j \leq n$ let $\tilde{\Delta}_{j} \subset \mathbb{R}^{n} \oplus \mathbb{R}^{1}$ be the graph of $\left.f_{j}\right|_{\Delta_{j}}$. For a subset $I \subset\{0,1, \ldots, n\}$ set $\tilde{\Delta}_{I}=\sum_{j \in I} \tilde{\Delta}_{j}$ and let $l$ be the closed ray $\{0\} \times\{x \in$ $\mathbb{R} \mid x \leq 0\}$ in $\mathbb{R}^{n} \oplus \mathbb{R}^{1}$. Then for any $I \subset\{0,1, \ldots, n\}$ the Minkowski sum $\tilde{\Delta}_{\{0,1, \ldots, n\}}$ majorizes the one $\tilde{\Delta}_{I}$. For a face $\tilde{\Gamma} \prec \tilde{\Delta}_{\{0,1, \ldots, n\}}$ we denote by $\tilde{\Gamma}_{I}$ the corresponding face of $\tilde{\Delta}_{I}$ and by $\Gamma_{I} \subset \mathbb{R}^{n}$ the projection of $\tilde{\Gamma}_{I}+l \subset \mathbb{R}^{n} \oplus \mathbb{R}^{1}$ to $\mathbb{R}^{n}$. Then we have $\operatorname{dim}\left(\tilde{\Gamma}_{I}+l\right)=\operatorname{dim} \Gamma_{I}+1$. Note that for $0 \leq j \leq n$ the projection $\Gamma_{\{j\}}$ is a face of $\Delta_{j}$, and we have $\Gamma_{I}=\sum_{j \in I} \Gamma_{\{j\}}$ for any $I \subset\{0,1, \ldots, n\}$. Moreover we have the following lemma. For $0 \leq j \leq n$ let $\mathbb{L}\left(\Delta_{j}\right)$ be the linear subspace of $\mathbb{R}^{n}$ that is parallel to the affine span of $\Delta_{j}$. Denote by $f_{j}^{L}: \mathbb{L}\left(\Delta_{j}\right) \longrightarrow \mathbb{R}$ the restriction of the linear part of $f_{j}$ to $\mathbb{L}\left(\Delta_{j}\right)$. Let $S=\oplus_{j=0}^{n} \mathbb{L}\left(\Delta_{j}\right)^{*}$ be the set of $(n+1)$ tuples $\left(g_{0}, g_{1}, \ldots, g_{n}\right)$ of such linear functions. 
Lemma 3.5. There exists an open dense subset $S_{\text {gen }}$ of $S$ such that for any $\left(g_{0}, g_{1}, \ldots, g_{n}\right) \in S_{\text {gen }}$ we have: If the polynomials $f_{0}, f_{1}, \ldots, f_{n}: \mathbb{R}^{n} \longrightarrow \mathbb{R}$ satisfy $f_{j}^{L}=$ $g_{j}(0 \leq j \leq n)$ then for any bounded face $\tilde{\Gamma}$ of $\tilde{\Delta}_{\{0,1, \ldots, n\}}+l$, which is also a face of $\tilde{\Delta}_{\{0,1, \ldots, n\}}$, the corresponding faces $\Gamma_{\{0\}}, \Gamma_{\{1\}}, \ldots, \Gamma_{\{n\}}$ are transversal: $\operatorname{dim}\left(\sum_{j=0}^{n} \Gamma_{\{j\}}\right)=\sum_{j=0}^{n}$ $\operatorname{dim} \Gamma_{\{j\}}$.

Proof. If some faces $G_{0}, G_{1}, \ldots, G_{0}$ of $\Delta_{0}, \Delta_{1}, \ldots, \Delta_{n}$ correspond to the same bounded face $\tilde{\Gamma}$ of $\tilde{\Delta}_{\{0,1, \ldots, n\}}+l$, then there exists a linear function $f: \mathbb{R}^{n} \longrightarrow \mathbb{R}$ such that $\left.f\right|_{\mathbb{L}\left(G_{j}\right)}=$ $\left.f_{j}^{L}\right|_{\mathbb{L}\left(G_{j}\right)}$ for any $0 \leq j \leq n$. For each such $(n+1)$-tuple $\left(G_{0}, \ldots, G_{n}\right)$ of faces, this last condition gives a restriction to $\left(f_{0}^{L}, \ldots, f_{n}^{L}\right)$ and hence defines a linear subspace $S\left(G_{0}, \ldots, G_{n}\right)$ of $S$. Note that if $G_{0}, \ldots, G_{n}$ are not transversal, the codimension of $S\left(G_{0}, \ldots, G_{n}\right)$ is positive. So it suffices to set $S_{\text {gen }}$ to be the complement of the union of such $S\left(G_{0}, \ldots, G_{n}\right){ }^{\prime}$ s.

By this lemma, after changing the linear parts of $f_{0}, f_{1}, \ldots, f_{n}$ slightly, we may assume that for any bounded face $\tilde{\Gamma}$ of $\tilde{\Delta}_{\{0,1, \ldots, n\}}+l$, the corresponding faces $\Gamma_{\{0\}}, \Gamma_{\{1\}}, \ldots, \Gamma_{\{n\}}$ are transversal. Then by applying Lemma 3.4 to the case $\square=\tilde{\Delta}_{\{0,1, \ldots, n\}}$, $\square^{\prime}=\tilde{\Delta}_{\{0\} \sqcup J}(J \subset\{1,2, \ldots, n\})$ and the closed ray $l=\{0\} \times\{x \in \mathbb{R} \mid x \leq 0\}$, we obtain

$$
\sum_{J \subset\{1,2, \ldots, n\}}(-1)^{\sharp J} \downarrow\left(\Delta_{\{0\} \sqcup J}\right)=\sum_{\tilde{\Gamma}}(-1)^{\operatorname{dim} \tilde{\Gamma}} \sum_{J \subset\{1,2, \ldots, n\}}(-1)^{\sharp J} \sharp\left(\Gamma_{\{0\} \sqcup J}\right),
$$

where $\tilde{\Gamma}$ ranges through the bounded faces of $\tilde{\Delta}_{\{0,1, \ldots, n\}}+l$. By the transversality of $\Gamma_{\{0\}}, \Gamma_{\{1\}}, \ldots, \Gamma_{\{n\}}$ for $\tilde{\Gamma} \prec \tilde{\Delta}_{\{0,1, \ldots, n\}}$ there are only the following two cases:

(a) There exists $1 \leq j \leq n$ such that $\operatorname{dim} \Gamma_{\{j\}}=0$.

(b) $\Gamma_{\{1\}}, \ldots, \Gamma_{\{n\}}$ are linearly independent segments and $\operatorname{dim} \Gamma_{\{0\}}=0$.

In the case (a), by applying Step (A) to $\Gamma_{\{0\}}, \Gamma_{\{1\}}, \ldots, \Gamma_{\{n\}}$ (the vertices of $\Gamma_{\{1\}}, \ldots, \Gamma_{\{n\}}$ are those of $\Delta_{1}, \ldots, \Delta_{n}$ and hence in $L$ ) we have

$$
\sum_{J \subset\{1,2, \ldots, n\}}(-1)^{n-\sharp J} \sharp\left(\Gamma_{\{0\} \sqcup J}\right)=0 .
$$

In particular, this is the case whenever $\operatorname{dim} \tilde{\Gamma}<n$. Moreover, in the case (b), by Step (B) we have

$$
\sum_{J \subset\{1,2, \ldots, n\}}(-1)^{n-\sharp J} \sharp\left(\Gamma_{\{0\} \sqcup J}\right)=\operatorname{MV}\left(\Gamma_{\{1\}}, \ldots, \Gamma_{\{n\}}\right) .
$$


Hence we get

$$
\begin{aligned}
& \sum_{\tilde{\Gamma}}(-1)^{\operatorname{dim} \tilde{\Gamma}} \sum_{J \subset\{1,2, \ldots, n\}}(-1)^{\sharp J} \sharp\left(\Gamma_{\{0\} \sqcup J}\right) \\
& =\sum_{\tilde{\Gamma}, \operatorname{dim} \tilde{\Gamma}=n}(-1)^{n} \sum_{J \subset\{1,2, \ldots, n\}}(-1)^{\sharp J} \sharp\left(\Gamma_{\{0\} \sqcup J}\right) \\
& =\sum_{\tilde{\Gamma}, \operatorname{dim} \tilde{\Gamma}=n} \operatorname{MV}\left(\Gamma_{\{1\}}, \ldots, \Gamma_{\{n\}}\right) .
\end{aligned}
$$

By reversing the arguments used to obtain (39) and (42) in the absense of the 0th polytopes $\Delta_{0}, \Gamma_{\{0\}}$ etc., we find that the last term of (42) is equal to

$$
\sum_{J \subset\{1,2, \ldots, n\}}(-1)^{\sharp J} \bigsqcup\left(\Delta_{J}\right)=\operatorname{MV}\left(\Delta_{1}, \ldots, \Delta_{n}\right) .
$$

Similarly we have

$$
\begin{aligned}
& \sum_{J \subset\{1,2, \ldots, n\}}(-1)^{n-\sharp J} \sharp\left(\Delta_{\{0\} \sqcup J}\right) \\
= & \sum_{\tilde{\Gamma}}(-1)^{\operatorname{dim} \tilde{\Gamma}} \sum_{J \subset\{1,2, \ldots, n\}}(-1)^{n-\sharp J} \downarrow\left(\Gamma_{\{0\} \sqcup J}\right) \\
= & \sum_{\tilde{\Gamma}, \operatorname{dim} \tilde{\Gamma}=n} \sum_{J \subset\{1,2, \ldots, n\}}(-1)^{\sharp J} \downarrow\left(\Gamma_{\{0\} \sqcup J}\right) \\
= & \sum_{\tilde{\Gamma}, \operatorname{dim} \tilde{\Gamma}=n} \operatorname{MV}\left(\Gamma_{\{1\}}, \ldots, \Gamma_{\{n\}}\right)=\operatorname{MV}\left(\Delta_{1}, \ldots, \Delta_{n}\right) .
\end{aligned}
$$

This completes the proof.

Now let us return to the situation in Proposition 2.12 and use the notations there. Then by applying Proposition 3.2 to the case $\Delta_{1}=\cdots=\Delta_{n}=\Delta, \Delta_{0}=\{-w(\alpha)\}$ and $L=L_{\tau}$, we obtain the following very simple result. We define a finite subset $\Lambda \subset \mathbb{C}$ by $\Lambda=\left\{\tau^{v} \mid v \in \mathbb{Z}^{n}\right\}$. 
Theorem 3.6. In the situation above, we have

$$
\chi\left(Z^{*}\right)_{\alpha}=\sum_{p, q} e^{p, q}\left(Z^{*}\right)_{\alpha}= \begin{cases}(-1)^{n-1} \frac{1}{\sharp \Lambda} \operatorname{Vol}_{\mathbb{Z}}(\Delta) & (\alpha \in \Lambda), \\ 0 & (\alpha \notin \Lambda),\end{cases}
$$

where $\operatorname{Vol}_{\mathbb{Z}}(*) \in \mathbb{Z}$ is the normalized $n$-dimensional volume with respect to the lattice $\mathbb{Z}^{n}$.

The following definition will be frequently used throughout this paper.

Definition 3.7. For a subvariety $Y^{*} \subset\left(\mathbb{C}^{*}\right)^{n}$ that is invariant by $l_{\tau}:\left(\mathbb{C}^{*}\right)^{n} \stackrel{\sim}{\longrightarrow}\left(\mathbb{C}^{*}\right)^{n}, p, q \geq$ 0 and $\alpha \in \mathbb{C}$ we define the virtual Betti polynomial $\beta\left(Y^{*}\right)_{\alpha}=\sum_{i=0}^{+\infty} \beta_{i}\left(Y^{*}\right)_{\alpha} \cdot t^{i} \in \mathbb{Z}[t]$ by $\beta_{i}\left(Y^{*}\right)_{\alpha}=\sum_{p+q=i} e^{p, q}\left(Y^{*}\right)_{\alpha} \in \mathbb{Z}$.

Definition 3.8. Let $\Delta$ be an $n$-dimensional integral polytope in $\left(\mathbb{R}^{n}, \mathbb{Z}^{n}\right)$.

(1) (see [4, Section 2.3]) We say that $\Delta$ is prime if for any vertex $w$ of $\Delta$ the cone $\operatorname{Con}(\Delta, w)$ is generated by a basis of $\mathbb{R}^{n}$.

(2) We say that $\Delta$ is pseudo-prime if for any 1-dimensional face $\gamma \prec \Delta$ the number of the two-dimensional faces $\gamma^{\prime} \prec \Delta$ such that $\gamma \prec \gamma^{\prime}$ is $n-1$.

By definition, prime polytopes are pseudo-prime. Moreover, for a pseudo-prime polytope $\Delta$, the projective toric variety $X_{\Delta}$ associated to the dual fan of $\Delta$ is an orbifold outside finitely many points. This implies that the closure of a nondegenerate hypersurface $Z^{*} \subset\left(\mathbb{C}^{*}\right)^{n}$ in $X_{\Delta}$ is quasi-smooth in the sense of [4] and has the Poincaré duality. By Matsui et al. [29, Corollary 2.15] and the proof of Theorem 3.6 we obtain the following proposition.

Proposition 3.9. In the situation of Proposition 2.12, assume moreover that the $n$-dimensional polytope $\Delta=\mathrm{NP}(g)$ is pseudo-prime. Then for any $\alpha \in \mathbb{C} \backslash\{1\}$ and $r \geq 0$, we have

$$
\beta_{r}\left(Z^{*}\right)_{\alpha}=(-1)^{n+r} \sum_{\substack{\Gamma<\Delta \\ \operatorname{dim} \Gamma=r+1}}\left\{\sum_{\gamma \prec \Gamma}(-1)^{\operatorname{dim} \gamma} \frac{1}{\sharp \Lambda(\gamma)} \operatorname{Vol}_{\mathbb{Z}}(\gamma)_{\alpha}\right\},
$$


where $\Lambda(\gamma) \subset \Lambda$ is defined similarly to $\Lambda$ by using the intersection of $\mathbb{Z}^{n}$ and the affine span of $\gamma$, and we define the integer $\operatorname{Vol}_{\mathbb{Z}}(\gamma)_{\alpha} \in \mathbb{Z}_{+}$by

$$
\operatorname{Vol}_{\mathbb{Z}}(\gamma)_{\alpha}= \begin{cases}\operatorname{Vol}_{\mathbb{Z}}(\gamma) & (\alpha \in \Lambda(\gamma)), \\ 0 & (\alpha \notin \Lambda(\gamma)) .\end{cases}
$$

Remark 3.10. By Proposition 3.9, we can rewrite the main results of [29] much more simply in terms of the volumes of polytopes (see [29, Theorem 5.9] etc.). In particular, by rewriting the results in [29, Section 7] for the case $k=1$, we obtain a combinatorial description of the numbers of the Jordan blocks in the (classical) Milnor monodromy $\Phi_{n-1,0}$. This result is different from the one previously obtained by Danilov [3] since we generalized the results in [4] to pseudo-prime polytopes.

Now let $\square \subset \mathbb{R}^{n}$ be an (n-1)-dimensional integral polytope whose affine span $K \simeq \mathbb{R}^{n-1}$ in $\mathbb{R}^{n}$ does not contain the origin $0 \in \mathbb{R}^{n}$. Denote by $\Delta$ the pyramid over $\square$ with apex $0 \in \mathbb{R}^{n}$ and let $d_{\square}>0$ be the lattice distance of $\square$ from $0 \in \mathbb{R}^{n}$. Let $Z^{*} \subset\left(\mathbb{C}^{*}\right)^{n}$ be a nondegenerate hypersurface whose Newton polytope is $\Delta$. Assume also that the support of the defining Laurent polynomial of $Z^{*}$ is contained in $\{0\} \sqcup \square$. Then we can define an automorphism of $Z^{*}$ of order $d_{\square}$ as follows. Let $\mathrm{ht}(*, K): \mathbb{R}^{n} \longrightarrow \mathbb{R}$ be the linear map such that $\operatorname{ht}(v, K)=d_{\square}>0$ for any $v \in K$. Then to the group homomorphism $\mathbb{Z}^{n} \longrightarrow \mathbb{C}^{*}$ defined by

$$
v \longmapsto \exp \left(2 \pi \sqrt{-1} \cdot \operatorname{ht}(v, K) / d_{\square}\right)
$$

we can naturally associate an element $\tau_{\square} \in\left(\mathbb{C}^{*}\right)^{n}=\operatorname{Spec}\left(\mathbb{C}\left[\mathbb{Z}^{n}\right]\right)$ such that $\left(\tau_{\square}\right)^{d}=1$. By construction $Z^{*} \subset\left(\mathbb{C}^{*}\right)^{n}$ is invariant by the multiplication by $\tau_{\square}$. Now fix a complex number $\alpha \neq 1$. Then by Theorem 3.6, the virtual Betti polynomial

$$
\beta(\square)_{\alpha}:=\beta\left(Z^{*}\right)_{\alpha} \in \mathbb{Z}[t]
$$

(of degree $\leq \operatorname{dim} \square=n-1$ ) of the hypersurface $Z^{*} \subset\left(\mathbb{C}^{*}\right)^{n}$ defined by the $(n-1)$ dimensional polytope $\square$ can be calculated as follows. First, for each face $\Gamma$ of $\square$, we define a polynomial $\beta(\Gamma)_{\alpha} \in \mathbb{Z}[t]$ of degree $\leq \operatorname{dim} \Gamma$ similarly. By induction on $\operatorname{dim} \square$, we may assume that for any proper face $\Gamma$ of $\square$ the polynomial $\beta(\Gamma)_{\alpha}$ is already determined. Let $\square^{\prime}$ be an (n-1)-dimensional prime integral polytope that majorizes $\square$ in the affine 
span $K$ of $\square$. For a face $\Gamma^{\prime}$ of $\square^{\prime}$, we denote by $\Gamma$ the corresponding face of $\square$. Then we can (uniquely) determine $\beta(\square)_{\alpha} \in \mathbb{Z}[t]$ by the following three conditions:

(i) The degree of $\beta(\square)_{\alpha}$ is $\leq \operatorname{dim} \square$.

(ii) The coefficients $c_{i}$ of the polynomial

$$
\sum_{i=0}^{+\infty} c_{i} t^{i}=\sum_{\Gamma^{\prime}<\square^{\prime}}\left(t^{2}-1\right)^{\operatorname{dim} \Gamma^{\prime}-\operatorname{dim} \Gamma} \beta(\Gamma)_{\alpha} \in \mathbb{Z}[t]
$$

are symmetric with respect to the degree $\operatorname{dim} \square: c_{\operatorname{dim} \square+k}=c_{\operatorname{dim} \square-k}$ for any $k \in \mathbb{Z}$.

(iii) $\beta(\square)_{\alpha}(1)=(-1)^{\operatorname{dim} \square} \operatorname{Vol}_{\mathbb{Z}}(\square)_{\alpha}$, where we set

$$
\operatorname{Vol}_{\mathbb{Z}}(\square)_{\alpha}= \begin{cases}\operatorname{Vol}_{\mathbb{Z}}(\square) \in \mathbb{Z}_{+} & \left(\alpha^{d_{\square}}=1\right), \\ 0 & \text { (otherwise) }\end{cases}
$$

Indeed, let $\Delta^{\prime}$ be the pyramid over $\square^{\prime}$ with apex $0 \in \mathbb{R}^{n}$ and $X_{\Delta^{\prime}}$ the projective toric variety associated to its dual fan. Note that $\Delta^{\prime}$ is pseudo-prime and majorizes $\Delta$. Then the closure of $Z^{*} \subset\left(\mathbb{C}^{*}\right)^{n}$ in $X_{\Delta^{\prime}}$ has the Poincaré duality, and (as in [4, Section 5.2]) by using Theorem 3.6 we obtain the above algorithm for the computation of $\beta(\square)_{\alpha}$. The following definition will play a crucial role in the proof of our main results.

Definition 3.11. For a complex number $\alpha \neq 1$ let $\beta(\square)_{\alpha} \in \mathbb{Z}[t]$ be the polynomial of degree $\leq \operatorname{dim} \square$ as above. Then for $m \geq \operatorname{dim} \square$, we set

$$
\beta(\square, m)_{\alpha}=\left(t^{2}-1\right)^{m-\operatorname{dim} \square} \beta(\square)_{\alpha} \in \mathbb{Z}[t] .
$$

\section{Motivic Milnor Fibers over C.I. and their Virtual Betti Polynomials}

For $2 \leq k \leq n$ let

$$
W=\left\{f_{1}=\cdots=f_{k-1}=0\right\} \supset V=\left\{f_{1}=\cdots=f_{k-1}=f_{k}=0\right\}
$$

be complete intersection subvarieties of $\mathbb{C}^{n}$ such that $0 \in V$. Assume that $W$ and $V$ have isolated singularities at the origin $0 \in \mathbb{C}^{n}$. Then by a fundamental result of Hamm [16], the Milnor fiber $F_{0}$ of $g:=\left.f_{k}\right|_{W}: W \longrightarrow \mathbb{C}$ at the origin 0 satisfies the condition 
$H^{j}\left(F_{0} ; \mathbb{C}\right) \simeq 0(j \neq 0, n-k)$. Recall that the semisimple part of the monodromy operator $\Phi_{n-k, 0}: H^{n-k}\left(F_{0} ; \mathbb{C}\right) \simeq H^{n-k}\left(F_{0} ; \mathbb{C}\right)$ was determined by Oka [33, 34] and Kirillov [20] (see also [27] for some generalizations). Our objective here is to describe the Jordan normal form of $\Phi_{n-k, 0}: H^{n-k}\left(F_{0} ; \mathbb{C}\right) \simeq H^{n-k}\left(F_{0} ; \mathbb{C}\right)$ in terms of the Newton polyhedrons of $f_{1}, f_{2}, \ldots, f_{k}$. For this purpose, we shall use the theory of mixed Hodge modules due to Saito [38, 40]. Let $\psi_{f_{k}}^{p}:=\psi_{f_{k}}[-1]: \mathbf{D}_{c}^{b}\left(\mathbb{C}^{n}\right) \longrightarrow \mathbf{D}_{c}^{b}\left(f_{k}^{-1}(0)\right)$ be the shifted nearby cycle functor that preserves the perversity. Let $\mathcal{F} \in \mathbf{D}_{c}^{b}\left(\mathbb{C}^{n}\right)$ be the minimal extension of the perverse sheaf $\mathbb{C}_{W \backslash\{0\}}[n-k+1] \in \mathbf{D}_{c}^{b}\left(\mathbb{C}^{n} \backslash\{0\}\right)$ to $\mathbb{C}^{n}$. Then the perverse sheaf $\psi_{f_{k}}^{p}(\mathcal{F}) \in \mathbf{D}_{c}^{b}\left(f_{k}^{-1}(0)\right)$ on $f_{k}^{-1}(0)$ has the following decomposition with respect to the eigenvalues $\lambda \in \mathbb{C}^{*}$ of its monodromy automorphism:

$$
\psi_{f_{k}}^{p}(\mathcal{F})=\bigoplus_{\lambda \in \mathbb{C}^{*}} \psi_{f_{k}, \lambda}^{p}(\mathcal{F})
$$

(see [8] etc.). By Proposition 2.3 for any $\lambda \neq 1$, the support of the perverse sheaf $\psi_{f_{k}, \lambda}^{p}(\mathcal{F})$ is contained in the origin $0 \in \mathbb{C}^{n}$. So we may regard $\psi_{f_{k}, \lambda}^{p}(\mathcal{F})(\lambda \neq 1)$ simply as complex vector spaces endowed with monodromy automorphisms. Now by using the mixed Hodge module over the perverse sheaf $\mathcal{F} \in \mathbf{D}_{c}^{b}\left(\mathbb{C}^{n}\right)$, to $\psi_{f_{k}}^{p}(\mathcal{F})_{0} \in \mathbf{D}_{c}^{b}(\{0\})$ and the semisimple part of its monodromy automorphism, we associate naturally an element

$$
\left[H_{g}\right] \in \mathrm{K}_{0}\left(\mathrm{HS}^{\mathrm{mon}}\right)
$$

(see Saito [38, 40] for the details). Then by construction, for any $\lambda \neq 1$ the $\lambda$-eigenspace part $\left[H_{g}\right]_{\lambda} \in \mathrm{K}_{0}(\mathrm{HS})$ of $\left[H_{g}\right] \in \mathrm{K}_{0}\left(\mathrm{HS}^{\mathrm{mon}}\right)$ is identified with the complex vector space $\psi_{f_{k}, \lambda}^{p}(\mathcal{F})$ endowed with a Hodge decomposition whose weights are defined by its "absolute" monodromy filtration (see Saito [38, 40]). Here we essentially used the purity of the mixed Hodge module over the perverse sheaf $\mathcal{F} \in \mathbf{D}_{c}^{b}\left(\mathbb{C}^{n}\right)$. For an element $[H] \in \mathrm{K}_{0}\left(\mathrm{HS}^{\mathrm{mon}}\right)$, $H \in \mathrm{HS}^{\text {mon }}$ with a quasi-unipotent endomorphism $\Psi: H \stackrel{\sim}{\longrightarrow} H, p, q \geq 0$ and $\lambda \in \mathbb{C}$ denote by $e^{p, q}([H])_{\lambda}$ the dimension of the $\lambda$-eigenspace of the morphism $H^{p, q} \stackrel{\sim}{\longrightarrow} H^{p, q}$ induced by $\Psi$ on the $(p, q)$-part $H^{p, q}$ of $H$. Then the following results are immediate consequences of the above construction and Saito's very deep theory in [38, 40]. Indeed, we can check the assertion (1) below by explicitly calculating the mixed Hodge numbers of our motivic Milnor fiber $\mathcal{S}_{g, 0}$.

Proposition 4.1. Assume that $\lambda \in \mathbb{C}^{*} \backslash\{1\}$. Then

(1) We have $e^{p, q}\left(\left[H_{g}\right]\right)_{\lambda}=0$ for $(p, q) \notin[0, n-k] \times[0, n-k]$. Moreover for $(p, q) \in$ $[0, n-k] \times[0, n-k]$ we have

$$
e^{p, q}\left(\left[H_{g}\right]\right)_{\lambda}=e^{n-k-q, n-k-p}\left(\left[H_{g}\right]\right)_{\lambda} .
$$


(2) For $i \geq 1$, the number of the Jordan blocks for the eigenvalue $\lambda$ with sizes $\geq i$ in $\Phi_{n-k, 0}: H^{n-k}\left(F_{0} ; \mathbb{C}\right) \stackrel{\sim}{\longrightarrow} H^{n-k}\left(F_{0} ; \mathbb{C}\right)$ is equal to

$$
\sum_{p+q=n-k-1+i, n-k+i} e^{p, q}\left(\left[H_{g}\right]\right)_{\lambda}
$$

Remark 4.2. By Proposition 2.3, for $\lambda=1$ the dimension of the support of $\psi_{f_{k}, \lambda}^{p}(\mathcal{F})$ is not zero in general. Therefore for $\lambda=1$ we cannot prove the symmetry of weights of $\left[H_{g}\right]_{\lambda} \in \mathrm{K}_{0}(\mathrm{HS})$ as in Proposition 4.1(1) (indeed we can easily find counterexamples). This fact explains the reason why the results on the Jordan blocks for the eigenvalue 1 in $\Phi_{n-k, 0}$ cannot be obtained by our methods. For related problems, see also for example Ebeling and Steenbrink [9].

By Proposition 4.1, for $\lambda \neq 1$ the calculation of the eigenvalue $\lambda$ part of the Jordan normal form of $\Phi_{n-k, 0}$ is reduced to that of $e^{p, q}\left(\left[H_{g}\right]\right)_{\lambda}$. Moreover, as in Denef and Loeser [5, 6] and Guibert-Loeser-Merle [15], by using a resolution of singularities of $W$ and $g: W \longrightarrow \mathbb{C}$ we can construct a motivic Milnor fiber $\mathcal{S}_{g, 0}$ of $g$ at $0 \in \mathbb{C}^{n}$, which enables us to calculate $e^{p, q}\left(\left[H_{g}\right]\right)_{\lambda}$ as follows. Let $\pi: X \longrightarrow \mathbb{C}^{n}$ be a proper morphism from a smooth algebraic variety $X$ such that $\left.\pi\right|_{X \backslash \pi^{-1}(0)}: X \backslash \pi^{-1}(0) \longrightarrow \mathbb{C}^{n} \backslash\{0\}$ is an isomorphism and $\pi^{-1}(0)=D_{1} \cup \cdots \cup D_{m}$ is a normal crossing divisor $\left(D_{1}, \ldots, D_{m}\right.$ are smooth) in $X$. Then via the isomorphism $X \backslash \pi^{-1}(0) \simeq \mathbb{C}^{n} \backslash\{0\}$, we regard $W \backslash\{0\}$ as a subset of $X$ and denote by $W^{\prime}$ its closure in $X$. We call $W^{\prime}$ the proper transform of $W$ in $X$. By Hironaka's theorem we can take $\pi: X \longrightarrow \mathbb{C}^{n}$ such that $W^{\prime}$ is smooth and intersects $D_{I}:=\bigcap_{i \in I} D_{i}$ transversally for any subset $I \subset\{1,2, \ldots, m\}$. We may assume also that the hypersurface $S:=\overline{f_{k}^{-1}(0) \backslash\{0\}} \subset X$ is smooth in a neighborhood of $W^{\prime}$ and intersects $D_{I} \cap W^{\prime}$ transversally for any $I \subset\{1,2, \ldots, m\}$. For $1 \leq i \leq m$ let $d_{i}>0$ be the order of the zero of $f_{k} \circ \pi$ along $D_{i}$. For a nonempty subset $I \subset\{1,2, \ldots, m\}$ set $D_{I}^{\circ}=D_{I} \backslash\left(\bigcup_{i \notin I} D_{i}\right)$,

$$
E_{I}^{\circ}=\left(D_{I}^{\circ} \cap W^{\prime}\right) \backslash S, \quad F_{I}^{\circ}=\left(D_{I}^{\circ} \cap W^{\prime}\right) \cap S
$$

and $d_{I}=\operatorname{gcd}\left(d_{i}\right)_{i \in I}>0$. Then, as in [6, Section 3.3], we can construct an unramified Galois covering $\widetilde{E_{I}^{\circ}} \longrightarrow E_{I}^{\circ}$ of $E_{I}^{\circ}$ as follows. First, let $U \subset X \backslash S$ be an affine open subset such that $f_{k} \circ \pi=h_{1, W} \cdot\left(h_{2, W}\right)^{d_{I}}$ on $U$, where $h_{1, W}$ is a unit on $U$ and $h_{2, W}: U \longrightarrow \mathbb{C}$ is a regular function. It is easy to see that $E_{I}^{\circ}$ is covered by such open subsets $U$. Then by gluing 
the varieties

$$
\left\{(t, x) \in \mathbb{C}^{*} \times\left(E_{I}^{\circ} \cap U\right) \mid h_{1, W}(x) \cdot t^{d_{I}}-1=0\right\}
$$

together in an obviously way, we obtain the $d_{I}$-fold covering $\widetilde{E_{I}^{\circ}}$ of $E_{I}^{\circ}$. Now for $d \in \mathbb{Z}_{>0}$, let $\mu_{d} \simeq \mathbb{Z} / \mathbb{Z} d$ be the multiplicative group consisting of the $d$ roots of unity $\left\{1, \zeta_{d}, \zeta_{d}^{2}, \ldots, \zeta_{d}^{d-1}\right\}$, where we set $\zeta_{d}:=\exp (2 \pi \sqrt{-1} / d) \in \mathbb{C}$. Then the unramified Galois covering $\widetilde{E_{I}^{\circ}}$ of $E_{I}^{\circ}$ admits a natural action of $\mu_{d_{I}}$ defined by assigning the automor$\operatorname{phism}(t, x) \longmapsto\left(\zeta_{d_{I}} t, x\right)$ of ${\widetilde{E_{I}^{\circ}}}^{\circ}$ to the generator $\zeta_{d_{I}} \in \mu_{d_{I}}$. Moreover, let $\hat{\mu}$ be the projective limit $\lim _{d} \mu_{d}$ of the projective system $\left\{\mu_{i}\right\}_{i \geq 1}$ with morphisms $\mu_{i d} \longrightarrow \mu_{i}$ given by $t \longmapsto t^{d}$. Then the variety $\widetilde{E_{I}^{\circ}}$ is endowed with a good $\hat{\mu}$-action in the sense of [6, Section 2.4]. Following the notations in [6], denote by $\mathcal{M}_{\mathbb{C}}^{\hat{\mu}}$ the ring obtained from the Grothendieck ring $K_{0}^{\hat{\mu}}\left(\operatorname{Var}_{\mathbb{C}}\right)$ of varieties over $\mathbb{C}$ with good $\hat{\mu}$-actions by inverting the Lefschetz motive $\mathbb{L} \simeq \mathbb{C} \in K_{0}^{\hat{\mu}}\left(\operatorname{Var}_{\mathbb{C}}\right)$. Recall that $\mathbb{L} \in K_{0}^{\hat{\mu}}\left(\operatorname{Var}_{\mathbb{C}}\right)$ is endowed with the trivial action of $\hat{\mu}$. We denote by $\left[\widetilde{E_{I}^{\circ}}\right]\left(\right.$ resp. $\left.\left[F_{I}^{\circ}\right]\right)$ the class of the variety $\widetilde{E_{I}^{\circ}}\left(\right.$ resp. $\left.F_{I}^{\circ}\right)$ endowed with the above $\hat{\mu}$-action (resp. the trivial $\hat{\mu}$-action) in $\mathcal{M}_{\mathbb{C}}^{\hat{\mu}}$.

Definition $4.3([5,6,15])$. We define the motivic Milnor fiber $\mathcal{S}_{g, 0} \in \mathcal{M}_{\mathbb{C}}^{\hat{\mu}}$ of $g: W \longrightarrow \mathbb{C}$ at the origin $0 \in \mathbb{C}^{n}$ by

$$
\mathcal{S}_{g, 0}=\sum_{I \neq \emptyset}\left\{(1-\mathbb{L})^{\sharp I-1}\left[\widetilde{E_{I}^{\circ}}\right]+(1-\mathbb{L})^{\sharp I}\left[F_{I}^{\circ}\right]\right\} \in \mathcal{M}_{\mathbb{C}}^{\hat{\mu}} .
$$

For the description of the element $\left[H_{g}\right] \in \mathrm{K}_{0}\left(\mathrm{HS}^{\mathrm{mon}}\right)$ in terms of $\mathcal{S}_{g, 0} \in \mathcal{M}_{\mathbb{C}}^{\hat{\mu}}$ let

$$
\chi_{h}: \mathcal{M}_{\mathbb{C}}^{\hat{\mu}} \longrightarrow \mathrm{K}_{0}\left(\mathrm{HS}^{\mathrm{mon}}\right)
$$

be the Hodge characteristic map defined in [6]. To a variety $Z$ with a good $\mu_{d}$-action, it associates the Hodge structure

$$
\chi_{h}([Z])=\sum_{j \in \mathbb{Z}}(-1)^{j}\left[H_{c}^{j}(Z ; \mathbb{Q})\right] \in \mathrm{K}_{0}\left(\mathrm{HS}^{\mathrm{mon}}\right)
$$

with the actions induced by the one $z \longmapsto \zeta_{d} \cdot z(z \in Z)$ on $Z$. Then by the proof of Denef and Loeser [5, Theorem 4.2.1], we obtain the following result. 
Theorem 4.4. In the Grothendieck group $K_{0}\left(\mathrm{HS}^{\mathrm{mon}}\right)$, we have

$$
\left[H_{g}\right]=(-1)^{n-k} \chi_{h}\left(\mathcal{S}_{g, 0}\right)
$$

Thus our problem was reduced to the calculation of $\chi_{h}\left(\mathcal{S}_{g, 0}\right) \in \mathrm{K}_{0}\left(\mathrm{HS}^{\mathrm{mon}}\right)$.

Definition 4.5. Let $f(x) \in \mathbb{C}\left[x_{1}, \ldots, x_{n}\right]$ be a polynomial on $\mathbb{C}^{n}$.

(1) We call the convex hull of $\bigcup_{v \in \operatorname{supp} f}\left\{v+\mathbb{R}_{+}^{n}\right\}$ in $\mathbb{R}_{+}^{n}$ the Newton polyhedron of $f$ at the origin $0 \in \mathbb{C}^{n}$ and denote it by $\Gamma_{+}(f)$.

(2) We say that $f$ is convenient if $\Gamma_{+}(f)$ intersects each coordinate axis of $\mathbb{R}^{n}$ outside the origin.

From now on, in order to describe our results explicitly, assume also that $f_{1}, f_{2}, \ldots, f_{k}$ are convenient. Set $f:=\left(f_{1}, f_{2}, \ldots, f_{k}\right)$ and

$$
\Gamma_{+}(f):=\Gamma_{+}\left(f_{1}\right)+\Gamma_{+}\left(f_{2}\right)+\cdots+\Gamma_{+}\left(f_{k}\right) .
$$

We denote the union of compact faces of $\Gamma_{+}(f)$ by $\Gamma_{f}$. Recall that on $\mathbb{R}_{+}^{n}$ we can define an equivalence relation by $u \sim u^{\prime} \Longleftrightarrow$ the supporting faces of $u$ and $u^{\prime}$ in $\Gamma_{+}(f)$ are the same. Then we obtain a decomposition $\mathbb{R}_{+}^{n}=\bigsqcup_{\Theta<\Gamma_{+}(f)} \sigma_{\Theta}$ of $\mathbb{R}_{+}^{n}$ into locally closed cones $\sigma_{\Theta}$. Since for a face $\Theta \prec \Gamma_{+}(f)$ such that $\Theta \subset \Gamma_{f}$ (i.e., a compact face $\Theta$ of $\left.\Gamma_{+}(f)\right)$ the supporting face of $u \in \sigma_{\Theta}$ in $\Gamma_{+}\left(f_{j}\right)$ does not depend on the choice of $u \in \sigma_{\Theta}$, we denote it simply by $\gamma_{j}^{\Theta}$. Then we have

$$
\Theta=\gamma_{1}^{\Theta}+\gamma_{2}^{\Theta}+\cdots+\gamma_{k}^{\Theta}
$$

For $1 \leq j \leq k$ and a compact face $\Theta$ of $\Gamma_{+}(f)$ we set

$$
f_{j}^{\Theta}(x)=\sum_{v \in \gamma_{j}^{\Theta} \cap \mathbb{Z}_{+}^{n}} a_{v} x^{v} \in \mathbb{C}\left[x_{1}, x_{2}, \ldots, x_{n}\right],
$$

where $f_{j}(x)=\sum_{v \in \mathbb{Z}_{+}^{n}} a_{v} x^{v}\left(a_{v} \in \mathbb{C}\right)$.

Definition 4.6 (see [34] etc.). We say that $f=\left(f_{1}, f_{2}, \ldots, f_{k}\right)$ is nondegenerate at the origin $0 \in \mathbb{C}^{n}$ if for any compact face $\Theta$ of $\Gamma_{+}(f)$ the two subvarieties $\left\{f_{1}^{\Theta}(x)=\cdots=\right.$ 
$\left.f_{k-1}^{\Theta}(x)=0\right\}$ and $\left\{f_{1}^{\Theta}(x)=\cdots=f_{k-1}^{\Theta}(x)=f_{k}^{\Theta}(x)=0\right\}$ in $\left(\mathbb{C}^{*}\right)^{n}$ are nondegenerate complete intersections.

From now on, let us assume also that $f$ is nondegenerate at the origin $0 \in$ $\mathbb{C}^{n}$. Then we can construct the morphism $\pi: X \longrightarrow \mathbb{C}^{n}$ explicitly as follows. Let $\Sigma_{1}=$ $\left\{\overline{\sigma_{\Theta}}\right\}_{\Theta<\Gamma_{+}(f)}$ be the dual fan of $\Gamma_{+}(f)$. Take a smooth subdivision $\Sigma$ of $\Sigma_{1}$ and denote by $X_{\Sigma}$ the smooth toric variety associated to the (smooth) fan $\Sigma$. We thus obtain a proper morphism $\pi: X_{\Sigma} \longrightarrow \mathbb{C}^{n}$ that induces an isomorphism $X_{\Sigma} \backslash \pi^{-1}(0) \simeq \mathbb{C}^{n} \backslash\{0\}$. Let $\rho_{1}, \rho_{2}, \ldots, \rho_{m}$ be the one-dimensional cones in $\Sigma$ such that $\rho_{i} \backslash\{0\} \subset \operatorname{Int}\left(\mathbb{R}_{+}^{n}\right)$ and for each $1 \leq i \leq m$ denote by $D_{i}$ the (smooth) toric divisor in $X_{\Sigma}$ that corresponds to $\rho_{i}$. Then we have $\pi^{-1}(0)=D_{1} \cup \cdots \cup D_{m}$ and it is a normal crossing divisor in $X_{\Sigma}$. Moreover, by the nondegeneracy of $f$, the proper transforms $W^{\prime}, S=\overline{f_{k}^{-1}(0) \backslash\{0\}}$ and $D_{i}$ 's satisfy the required smoothness and transversality. By using this explicit construction of $\pi: X_{\Sigma} \longrightarrow \mathbb{C}^{n}$, we can express the Hodge realizations $\chi_{h}\left(\mathcal{S}_{g, 0}\right)_{\lambda} \in \mathrm{K}_{0}(\mathrm{HS})(\lambda \neq 1)$ of our motivic Milnor fiber $\mathcal{S}_{g, 0}$ very concretely as follows. For a face $\Theta \prec \Gamma_{+}(f)$ such that $\Theta \subset \Gamma_{f}$ let $\mathbb{L}_{\Theta} \simeq \mathbb{R}^{\operatorname{dim} \Theta}$ be the linear subspace of $\mathbb{R}^{n}$ that is parallel to the affine span of $\Theta$. We denote by $K_{\Theta} \simeq \mathbb{R}^{\operatorname{dim} \Theta}$ the affine linear subspace of $\mathbb{R}^{n}$ that is parallel to $\mathbb{L}_{\Theta}$ and contains $\gamma_{k}^{\Theta}$ (see Figure 1). Let $\tilde{\mathbb{L}}_{\Theta} \simeq \mathbb{R}^{\operatorname{dim} \Theta+1}$ be the linear subspace of $\mathbb{R}^{n}$ generated by $\{0\} \sqcup K_{\Theta}$. Then $\mathbb{L}_{\Theta}$ is a hyperplane of $\tilde{\mathbb{L}}_{\Theta}$, and to the lattice $\tilde{M}_{\Theta}=\mathbb{Z}^{n} \cap \tilde{\mathbb{L}}_{\Theta}$ we can naturally associate the algebraic torus

$$
\tilde{T}_{\Theta}=\operatorname{Spec}\left(\mathbb{C}\left[\tilde{M}_{\Theta}\right]\right) \simeq\left(\mathbb{C}^{*}\right)^{\operatorname{dim} \Theta+1} .
$$

Denote the convex hull of $\{0\} \sqcup \gamma_{k}^{\Theta}$ in $\tilde{\mathbb{L}}_{\Theta}$ by $\Delta_{\gamma_{k}^{\Theta}}$ and for $1 \leq j \leq k-1$ let $\kappa_{j}^{\Theta}$ be an integral translation of $\gamma_{j}^{\Theta}$ in $\tilde{\mathbb{L}}_{\Theta}$ such that $\kappa_{j}^{\Theta} \subset K_{\Theta}$ (see Figure 1). For simplicity, we denote the $k$-tuple $\left(\kappa_{1}^{\Theta}, \ldots, \kappa_{k-1}^{\Theta}, \Delta_{\gamma_{k}^{\Theta}}\right)$ of integral polytopes in $\left(\tilde{\mathbb{L}}_{\Theta}, \tilde{M}_{\Theta}\right)$ by $\Delta_{\Theta}$. Let $d_{\Theta}>0$ be the lattice distance of the hyperplane $K_{\Theta} \subset \tilde{\mathbb{L}}_{\Theta}$ from the origin $0 \in \tilde{\mathbb{L}}_{\Theta}$. Note that $d_{\Theta}$ can be an integral multiple of the lattice distance $d\left(\gamma_{k}^{\Theta}\right)>0$ of $\gamma_{k}^{\Theta}$ from $0 \in \tilde{\mathbb{L}}_{\Theta}$ if $\operatorname{dim} \gamma_{k}^{\Theta}<\operatorname{dim} \Theta$. Then to $\Delta_{\Theta}$ we can naturally associate a nondegenerate complete intersection subvariety $Z_{\Delta_{\Theta}}^{*}$ of $\tilde{T}_{\Theta} \simeq\left(\mathbb{C}^{*}\right)^{\operatorname{dim} \Theta+1}$ and an action of the cyclic group $\mu_{d_{\Theta}}=\mathbb{Z} / \mathbb{Z} d_{\Theta}$ on it as follows. Let $g_{j}^{\Theta}(j=1,2, \ldots, k-1)$ and $\tilde{g}_{k}^{\Theta}$ be Laurent polynomials on $\tilde{T}_{\Theta}$ such that $\operatorname{NP}\left(g_{j}^{\Theta}\right)=\kappa_{j}^{\Theta}$ and $\operatorname{NP}\left(\tilde{g}_{k}^{\Theta}\right)=\Delta_{\gamma_{k}^{\Theta}}$. Assume also that the support supp $\tilde{g}_{k}^{\Theta}$ of $\tilde{g}_{k}^{\Theta}$ is contained in $\{0\} \sqcup \gamma_{k}^{\Theta}$ and the subvariety

$$
Z_{\Delta_{\Theta}}^{*}=\left\{g_{1}^{\Theta}(x)=\cdots=g_{k-1}^{\Theta}(x)=\tilde{g}_{k}^{\Theta}(x)=0\right\} \subset \tilde{T}_{\Theta}
$$

of $\tilde{T}_{\Theta}$ is a nondegenerate complete intersection. Let $h t\left(*, K_{\Theta}\right): \tilde{\mathbb{L}}_{\Theta} \longrightarrow \mathbb{R}$ be the linear map such that $\operatorname{ht}\left(v, K_{\Theta}\right)=d_{\Theta}>0$ for any $v \in K_{\Theta}$. Then to the group homomorphism $\tilde{M}_{\Theta} \longrightarrow \mathbb{C}^{*}$ 
defined by

$$
v \longmapsto \exp \left(2 \pi \sqrt{-1} \cdot \operatorname{ht}\left(v, K_{\Theta}\right) / d_{\Theta}\right)
$$

we can naturally associate an element $\tau_{\Theta} \in \tilde{T}_{\Theta}=\operatorname{Spec}\left(\mathbb{C}\left[\tilde{M}_{\Theta}\right]\right)$ such that $\left(\tau_{\Theta}\right)^{d_{\Theta}}=1$. Since $Z_{\Delta_{\Theta}}^{*} \subset \tilde{T}_{\Theta}$ is invariant by the multiplication $l_{\tau_{\Theta}}: \tilde{T}_{\Theta} \stackrel{\sim}{\longrightarrow} \tilde{T}_{\Theta}$ by $\tau_{\Theta}$, the variety $Z_{\Delta_{\Theta}}^{*}$ admits an action of $\mu_{d_{\Theta}}$. We thus obtain an element $\left[Z_{\Delta_{\Theta}}^{*}\right] \in \mathcal{M}_{\mathbb{C}}^{\hat{\mu}}$. Finally, for the compact face $\Theta \prec \Gamma_{+}(f)$, let $s_{\Theta}$ be the dimension of the minimal coordinate subspace of $\mathbb{R}^{n}$ containing $\Theta$ and set $m_{\Theta}=s_{\Theta}-\operatorname{dim} \Theta-1 \geq 0$.

Theorem 4.7. Assume that $\lambda \in \mathbb{C}^{*} \backslash\{1\}$. Then

(1) In the Grothendieck group $K_{0}(\mathrm{HS})$, we have

$$
\chi_{h}\left(\mathcal{S}_{g, 0}\right)_{\lambda}=\sum_{\Theta \subset \Gamma_{f}, \operatorname{dim} \Theta \geq k-1} \chi_{h}\left((1-\mathbb{L})^{m_{\Theta}} \cdot\left[Z_{\Delta_{\Theta}}^{*}\right)_{\lambda}\right.
$$

In particular, the virtual Betti polynomial $\beta\left(\mathcal{S}_{g, 0}\right)_{\lambda} \in \mathbb{Z}[t]$ is given by

$$
\beta\left(\mathcal{S}_{g, 0}\right)_{\lambda}=\sum_{\Theta \subset \Gamma_{f}, \operatorname{dim} \Theta \geq k-1}\left(1-t^{2}\right)^{m_{\Theta}} \cdot \beta\left(Z_{\Delta_{\Theta}}^{*}\right)_{\lambda}
$$

(2) For $i \geq 1$, the number of the Jordan blocks for the eigenvalue $\lambda$ with sizes $\geq i$ in $\Phi_{n-k, 0}: H^{n-k}\left(F_{0} ; \mathbb{C}\right) \simeq H^{n-k}\left(F_{0} ; \mathbb{C}\right)$ is equal to

$$
(-1)^{n-k}\left\{\beta_{n-k-1+i}\left(\mathcal{S}_{g, 0}\right)_{\lambda}+\beta_{n-k+i}\left(\mathcal{S}_{g, 0}\right)_{\lambda}\right\} .
$$

Proof. By using the above explicit construction of $\pi: X_{\Sigma} \longrightarrow \mathbb{C}^{n}$ from $\Gamma_{+}(f)$ the proof of (1) is obtained completely in the same way as that of [29, Theorems 5.3 and 7.3]. Then the assertion (2) follows immediately from Proposition 4.1(2).

By the Cayley trick in [4, Section 6], we can rewrite the formula for $\beta\left(\mathcal{S}_{g, 0}\right)_{\lambda} \in \mathbb{Z}[t]$ $(\lambda \neq 1)$ in Theorem 4.7(1) as follows. For a face $\Theta \prec \Gamma_{+}(f)$ such that $\Theta \subset \Gamma_{f}$, we define an open subset $\Omega_{\Theta}$ of $\tilde{T}_{\Theta} \times \mathbb{P}^{k-1}$ by

$$
\Omega_{\Theta}=\left\{\left(x ;\left(\alpha_{1}: \cdots: \alpha_{k}\right)\right) \in \tilde{T}_{\Theta} \times \mathbb{P}^{k-1} \mid \sum_{j=1}^{k-1} \alpha_{j} g_{j}^{\Theta}(x)+\alpha_{k} \tilde{g}_{k}^{\Theta}(x) \neq 0\right\} .
$$


By the standard decomposition $\mathbb{C}^{k}=\bigsqcup_{I \subset\{1,2, \ldots, k\}} T_{I}, T_{I} \simeq\left(\mathbb{C}^{*}\right)^{\sharp I}$ of $\mathbb{C}^{k}$, we obtain a stratification $\mathbb{P}^{k-1}=\bigsqcup_{I \neq \emptyset} \mathbb{P}\left(T_{I}\right)$ of $\mathbb{P}^{k-1}$, where we set

$$
\mathbb{P}\left(T_{I}\right)=\left\{\left(\alpha_{1}: \cdots: \alpha_{k}\right) \in \mathbb{P}^{k-1} \mid \alpha_{j}=0(j \notin I), \alpha_{j} \neq 0(j \in I)\right\} \simeq\left(\mathbb{C}^{*}\right)^{\sharp I-1} .
$$

For each subset $J \subset\{1,2, \ldots, k-1\}(J$ can be an empty set Ø), set

$$
\Omega_{\Theta, J}=\left\{\tilde{T}_{\Theta} \times \mathbb{P}\left(T_{J \sqcup\{k\}}\right)\right\} \cap \Omega_{\Theta} .
$$

Note that $\Omega_{\Theta, J}$ is the complement of the hypersurface

$$
Z_{\Theta, J}^{*}=\left\{\left(x ; \alpha_{j}(j \in J)\right) \in \tilde{T}_{\Theta} \times\left(\mathbb{C}^{*}\right)^{\sharp J} \mid \sum_{j \in J} \alpha_{j} g_{j}^{\Theta}(x)+\tilde{g}_{k}^{\Theta}(x)=0\right\}
$$

of the algebraic torus $\tilde{T}_{\Theta} \times\left(\mathbb{C}^{*}\right)^{\sharp J}$. Since this hypersurface $Z_{\Theta, J}^{*}$ is invariant by the multiplication of $\left(\tau_{\Theta}, 1\right) \in \tilde{T}_{\Theta} \times\left(\mathbb{C}^{*}\right)^{\sharp J}$, we obtain an action of $\mu_{d_{\Theta}}$ on $Z_{\Theta, J}^{*}$ and an element $\left[Z_{\Theta, J}^{*}\right] \in \mathcal{M}_{\mathbb{C}}^{\hat{\mu}}$.

Lemma 4.8. For $\lambda \in \mathbb{C}^{*} \backslash\{1\}$ and a face $\Theta \prec \Gamma_{+}(f)$ such that $\Theta \subset \Gamma_{f}$ we have

$$
\beta\left(Z_{\Delta_{\Theta}}^{*}\right)_{\lambda}=\frac{1}{t^{2 k-2}} \sum_{J \subset\{1,2, \ldots, k-1\}} \beta\left(Z_{\Theta, J}^{*}\right)_{\lambda}
$$

Proof. By the definition of $Z_{\Delta_{\Theta}}^{*}$ the natural projection

$$
\pi: \Omega_{\Theta} \longrightarrow \tilde{T}_{\Theta} \backslash Z_{\Delta_{\Theta}}^{*}
$$

is an algebraic fiber bundle whose fiber is isomorphic to $\mathbb{C}^{k-1}$. Therefore by the condition $\lambda \neq 1$ we obtain

$$
\beta\left(\tilde{T}_{\Theta} \backslash Z_{\Delta_{\Theta}}^{*}\right)_{\lambda}=\frac{1}{t^{2 k-2}} \sum_{J \subset\{1,2, \ldots, k-1\}} \beta\left(\Omega_{\Theta, J}\right)_{\lambda}
$$

Since the multiplication of $\tau_{\Theta}$ on $\tilde{T}_{\Theta}$ (resp. $\left(\tau_{\Theta}, 1\right)$ on $\left.\tilde{T}_{\Theta} \times\left(\mathbb{C}^{*}\right)^{\sharp J}\right)$ is homotopic to the identity, by $\lambda \neq 1$ we obtain the desired formula. This completes the proof. 
First, by replacing the defining equations of $Z_{\Delta_{\Theta}}^{*}$ with the help of Lemma 2.13, we may assume that the hypersurfaces $Z_{\Theta, J}^{*} \subset \tilde{T}_{\Theta} \times\left(\mathbb{C}^{*}\right)^{\sharp J}$ are nondegenerate. For a face $\Theta \prec \Gamma_{+}(f)$ such that $\Theta \subset \Gamma_{f}$ and $J \subset\{1,2, \ldots, k-1\}$ we denote by $\Theta_{J}$ the convex hull of

$$
\left(\gamma_{k}^{\Theta} \times\{0\}\right) \sqcup\left\{\bigsqcup_{j \in J}\left(\kappa_{j}^{\Theta} \times\left\{e_{j}\right\}\right)\right\}
$$

in $\tilde{\mathbb{L}}_{\Theta} \times \mathbb{R}^{\sharp J}$, where $e_{j}=(0, \ldots, 0,1,0, \ldots, 0)$ is the $j$ th standard unit vector in $\mathbb{R}^{\sharp J}$. This integral polytope $\Theta_{J}$ is called the join of $\kappa_{j}^{\Theta}(j \in J)$ and $\gamma_{k}^{\Theta}$. Let $\tilde{\Theta}_{J}$ be the convex hull of $\{(0,0)\} \sqcup \Theta_{J}$ in $\tilde{\mathbb{L}}_{\Theta} \times \mathbb{R}^{\sharp J}$. Then by Definition 3.11, we have

$$
\beta\left(Z_{\Theta, J}^{*}\right)_{\lambda}=\beta\left(\Theta_{J}, \operatorname{dim} \Theta+\sharp J\right)_{\lambda}=\left(t^{2}-1\right)^{\operatorname{dim} \Theta+\sharp J-\operatorname{dim} \Theta_{J}} \beta\left(\Theta_{J}\right)_{\lambda}
$$

for any $\lambda \in \mathbb{C}^{*} \backslash\{1\}$. Recall that if $\lambda \neq 1$ the degree of the polynomial $\beta\left(\Theta_{J}\right)_{\lambda} \in \mathbb{Z}[t]$ is $\leq \operatorname{dim} \Theta_{J}=\operatorname{dim}\left(\gamma_{k}^{\Theta}+\sum_{j \in J} \kappa_{j}^{\Theta}\right)+\sharp J$. Then for $\lambda \in \mathbb{C}^{*} \backslash\{1\}$ we obtain

$$
\begin{aligned}
\left(1-t^{2}\right)^{m_{\Theta}} \beta\left(Z_{\Delta_{\Theta}}^{*}\right)_{\lambda} & =\frac{(-1)^{m_{\Theta}}}{t^{2 k-2}} \sum_{J \subset\{1,2, \ldots, k-1\}}\left(t^{2}-1\right)^{s_{\Theta}+\sharp J-1-\operatorname{dim} \Theta_{J}} \beta\left(\Theta_{J}\right)_{\lambda} \\
& =\frac{(-1)^{m_{\Theta}}}{t^{2 k-2}} \sum_{J \subset\{1,2, \ldots, k-1\}} \beta\left(\Theta_{J}, s_{\Theta}+\sharp J-1\right)_{\lambda} .
\end{aligned}
$$

Moreover for a nondegenerate hypersurface $S_{\Theta, J}^{*} \subset\left(\mathbb{C}^{*}\right)^{S_{\Theta}+\sharp J}$ having the thin Newton polytope $\tilde{\Theta}_{J} \subset \mathbb{R}^{s_{\Theta}+\sharp J}$ (recall that $\tilde{\mathbb{L}}_{\Theta} \subset \mathbb{R}^{s_{\Theta}}$ ) and a natural action of $\mu_{d_{\Theta}}$ on it, we have

$$
\beta\left(\Theta_{J}, S_{\Theta}+\sharp J-1\right)_{\lambda}=\beta\left(S_{\Theta, J}^{*}\right)_{\lambda}
$$

for any $\lambda \in \mathbb{C}^{*} \backslash\{1\}$. We thus obtain the following theorem.

Theorem 4.9. For $\lambda \in \mathbb{C}^{*} \backslash\{1\}$ we have

$$
\begin{aligned}
\beta\left(\mathcal{S}_{g, 0}\right)_{\lambda} & =\sum_{\Theta \subset \Gamma_{f}, \operatorname{dim} \Theta \geq k-1} \frac{(-1)^{m_{\Theta}}}{t^{2 k-2}} \sum_{J \subset\{1,2, \ldots, k-1\}} \beta\left(\Theta_{J}, s_{\Theta}+\sharp J-1\right)_{\lambda} \\
& =\sum_{\Theta \subset \Gamma_{f}, \operatorname{dim} \Theta \geq k-1} \frac{(-1)^{m_{\Theta}}}{t^{2 k-2}} \sum_{J \subset\{1,2, \ldots, k-1\}} \beta\left(S_{\Theta, J}^{*}\right)_{\lambda} .
\end{aligned}
$$


Remark 4.10. Although we formulated Theorem 4.9 for complete intersection singularities, it can be readily extended to the case of isolated determinantal singularities studied in [11] by constructing their toric resolutions. See [11] for the construction of toric resolutions of determinantal singularities.

By Theorems 4.7, 4.9 and the results in Sections 2 and 3, we can calculate the numbers of the Jordan blocks for the eigenvalues $\lambda \neq 1$ in $\Phi_{n-k, 0}: H^{n-k}\left(F_{0} ; \mathbb{C}\right) \simeq$ $H^{n-k}\left(F_{0} ; \mathbb{C}\right)$ as follows. First, for a compact face $\Theta \prec \Gamma_{+}(f)$ such that $\operatorname{dim} \Theta \geq k-1$ and a subset $J \subset\{1,2, \ldots, k-1\}$ we define an integer $c(\Theta, J) \in \mathbb{Z}_{+}$by $c(\Theta, J)=\operatorname{dim} \Theta-$ $\operatorname{dim}\left(\gamma_{k}^{\Theta}+\sum_{j \in J} \kappa_{j}^{\Theta}\right)$. Then we have $\operatorname{dim} \Theta_{J}=\operatorname{dim} \Theta-c(\Theta, J)+\sharp J$. Moreover for $l \geq 1$ we define a finite subset $R\left(\Theta_{J}, l\right) \subset\left[0, \operatorname{dim} \Theta_{J}\right] \cap \mathbb{Z}$ by

$$
R\left(\Theta_{J}, l\right)=\left\{0 \leq r \leq \operatorname{dim} \Theta_{J} \mid n+k-3+l \equiv r \bmod 2\right\}
$$

For each $r \in R\left(\Theta_{J}, l\right)$, we set

$$
e\left(\Theta_{J}, l\right)_{r}:=\frac{n+k-3+l-r}{2} \in \mathbb{Z}_{+}
$$

Now in the situation above, let $\lambda \in \mathbb{C}^{*} \backslash\{1\}$ and $i \geq 1$. Then by Theorems 4.7 and 4.9 , the number of the Jordan blocks for the eigenvalue $\lambda$ with sizes $\geq i$ in $\Phi_{n-k, 0}$ is

$$
\sum_{\substack{\Theta \subset \Gamma_{f}, \operatorname{dim} \Theta \geq k-1}} \sum_{J \subset\{1, \ldots, k-1\}}(-1)^{n-k+c(\Theta, J)}\left\{\sum_{l=i, i+1}\left(\sum_{r \in R\left(\Theta_{J}, l\right)}(-1)^{e\left(\Theta_{J}, l\right)_{r}}\left(\begin{array}{c}
m_{\Theta}+c(\Theta, J) \\
e\left(\Theta_{J}, l\right)_{r}
\end{array}\right) \beta_{r}\left(\Theta_{J}\right)_{\lambda}\right)\right\} .
$$

Note that we can always calculate the virtual Betti numbers $\beta_{r}\left(\Theta_{J}\right)_{\lambda} \in \mathbb{Z}$ by our algorithm at the end of Section 3. We can construct polytopes that majorize the join $\Theta_{J}$ much easier than that for arbitrary polytopes of the same dimension. From now on, assume moreover that for any compact face $\Theta \prec \Gamma_{+}(f)$ such that $\operatorname{dim} \Theta \geq k-1$ the corresponding faces $\gamma_{j}^{\Theta}(1 \leq j \leq k)$ are simplicial and transversal: $\operatorname{dim}\left(\sum_{j=1}^{k} \gamma_{j}^{\Theta}\right)=\sum_{j=1}^{k} \operatorname{dim} \gamma_{j}^{\Theta}$. Note that this condition was used in [10] to describe the difference of the Euler characteristics of two "real" Milnor fibers over real complete intersections. Under this condition, the join $\Theta_{J}$ is 
prime and hence $\tilde{\Theta}_{J}$ is pseudo-prime. Therefore, by Proposition 3.9 we have

$$
\begin{aligned}
\beta_{r}\left(\Theta_{J}\right)_{\lambda} & =(-1)^{\operatorname{dim} \Theta_{J}+r} \sum_{\substack{\Gamma<\Theta_{J}, \operatorname{dim} \Gamma=r}}\left\{\sum_{\gamma \prec \Gamma}(-1)^{\operatorname{dim} \gamma} \operatorname{Vol}_{\mathbb{Z}}(\gamma)_{\lambda}\right\} \\
& =(-1)^{\operatorname{dim} \Theta_{J}+r} \sum_{l=0}^{r}\left\{\sum_{\substack{\gamma<\Theta_{J}, \operatorname{dim} \gamma=l}}(-1)^{l}\left(\begin{array}{c}
\operatorname{dim} \Theta_{J}-l \\
r-l
\end{array}\right) \operatorname{Vol}_{\mathbb{Z}}(\gamma)_{\lambda}\right\},
\end{aligned}
$$

where by using the lattice distance $d(\gamma)>0$ of the face $\gamma \prec \Theta_{J}$ from the origin $(0,0) \in$ $\tilde{\mathbb{L}}_{\Theta} \times \mathbb{R}^{\sharp J}$ we define the integer $\operatorname{Vol}_{\mathbb{Z}}(\gamma)_{\lambda} \in \mathbb{Z}_{+}$by

$$
\operatorname{Vol}_{\mathbb{Z}}(\gamma)_{\lambda}= \begin{cases}\operatorname{Vol}_{\mathbb{Z}}(\gamma) & \left(\lambda^{d(\gamma)}=1\right) \\ 0 & \text { (otherwise) }\end{cases}
$$

Finally to end this section, we shall introduce an analogue of the Steenbrink conjecture proved by Varchenko-Khovanskii [48] and Saito [39].

Definition 4.11 (Ebeling and Steenbrink [9]). As a Puiseux series, we define the nonintegral part $\operatorname{sp}_{g}(t)$ of the spectrum of $g: W \longrightarrow \mathbb{C}$ at the origin $0 \in \mathbb{C}^{n}$ by

$$
\operatorname{sp}_{g}(t)=\sum_{b \in(0,1) \cap \mathbb{Q}}\left[\sum_{i=0}^{n-k}\left\{\sum_{q \geq 0} e^{i, q}\left(\left[H_{g}\right]\right)_{\exp (-2 \pi \sqrt{-1} b)}\right\} t^{i+b}\right]
$$

By Proposition 4.1(i) the support of $\operatorname{sp}_{g}(t)$ is contained in the open interval $(0, n-k+1)$ and has the symmetry

$$
\operatorname{sp}_{g}(t)=t^{n-k+1} \operatorname{sp}_{g}\left(\frac{1}{t}\right)
$$

with center at $\frac{n-k+1}{2}$. Moreover by the above arguments (the Cayley trick) and the proof of [29, Theorem 5.10], we immediately obtain the following explicit description of $\operatorname{sp}_{g}(t)$. For each $\Theta \prec \Gamma_{+}(f)$ such that $\Theta \subset \Gamma_{f}$ and $J \subset\{1,2, \ldots, k-1\}$ let $\operatorname{Cone}\left(\Theta_{J}\right)=\mathbb{R}_{+} \Theta_{J} \subset \tilde{\mathbb{L}}_{\Theta} \times$ $\mathbb{R}^{\sharp J}$ be the cone generated by $\Theta_{J}$ and $h_{\Theta, J}: \operatorname{Cone}\left(\Theta_{J}\right) \longrightarrow \mathbb{R}$ the linear function such that 
$\left.h_{\Theta, J}\right|_{\Theta_{J}} \equiv 1$. Then we define the Puiseux series $P_{\Theta, J}(t)$ by

$$
P_{\Theta, J}(t)=\sum_{b \in \mathbb{Q}_{+} \backslash \mathbb{Z}_{+}} \sharp\left\{v \in \operatorname{Cone}\left(\Theta_{J}\right) \cap \mathbb{Z}^{n+\sharp J} \mid h_{\Theta, J}(v)=b\right\} t^{b} .
$$

Theorem 4.12. In the situation above, we have

$$
\operatorname{sp}_{g}(t)=(-1)^{n-k} \sum_{\Theta \subset \Gamma_{f}, \operatorname{dim} \Theta \geq k-1}\left\{\sum_{J \subset\{1, \ldots, k-1\}}(-1)^{\operatorname{dim} \Theta+\sharp J}(1-t)^{S_{\Theta}+\sharp J} P_{\Theta, J}(t)\right\} \cdot t^{-k+1} .
$$

\section{The Numbers of Jordan Blocks in the Monodromies over C.I.}

In this section, by using the results in the previous sections, we prove some combinatorial formulas for the Jordan normal forms of the (local) monodromies over complete intersection subvarieties of $\mathbb{C}^{n}$. We inherit the situation and the notations in Section 4. Then our primary interest here is to describe the numbers of the maximal (and the second maximal) Jordan blocks for the eigenvalues $\lambda \neq 1$ in the monodromy $\Phi_{n-k, 0}$ : $H^{n-k}\left(F_{0} ; \mathbb{C}\right) \simeq H^{n-k}\left(F_{0} ; \mathbb{C}\right)$ in terms of the Newton polyhedrons $\Gamma_{+}\left(f_{1}\right), \Gamma_{+}\left(f_{2}\right), \ldots, \Gamma_{+}\left(f_{k}\right)$. We fix $\lambda \in \mathbb{C}^{*} \backslash\{1\}$ and a face $\Theta \prec \Gamma_{+}(f)$ such that $\Theta \subset \Gamma_{f}$. First, for $J \subset\{1,2, \ldots, k-1\}$ let $K_{\Theta, J}$ be the affine linear subspace of $\tilde{\mathbb{L}}_{\Theta} \simeq \mathbb{R}^{\operatorname{dim} \Theta+1}$, which is parallel to the affine span of the Minkowski sum $\gamma_{k}^{\Theta}+\sum_{j \in J} \kappa_{j}^{\Theta}$ and contains $\gamma_{k}^{\Theta}$. Then we define an integer $d_{\Theta, J}>0$ to be the lattice distance of $K_{\Theta, J}$ from the origin $0 \in \tilde{\mathbb{L}}_{\Theta}$. Note that if $J=\{1,2, \ldots, k-1\}$ (resp. $J=\emptyset$ ) $d_{\Theta, J}$ is equal to $d_{\Theta}$ (resp. is the lattice distance $d\left(\gamma_{k}^{\Theta}\right.$ ) of $\gamma_{k}^{\Theta}$ from the origin $\left.0 \in \tilde{\mathbb{L}}_{\Theta}\right)$. Moreover, for any $J \subset\{1,2, \ldots, k-1\}$ we see that $d_{\Theta, J}$ divides $d_{\Theta}$.

Definition 5.1. For $J \subset\{1,2, \ldots, k-1\}$ we denote the $\operatorname{difference} \operatorname{dim}\left(\gamma_{k}^{\Theta}+\sum_{j \in J} \kappa_{j}^{\Theta}\right)-$ $\sharp J$ by $\delta(\Theta, J)$.

The following lemma is essentially due to Sturmfels [43].

Lemma 5.2. If $\delta(\Theta, J) \geq 0$ for any $J \subset\{1,2, \ldots, k-1\}$, then the set $\{J \mid \delta(\Theta, J)=0\}$ is closed by unions $\cup$ and intersections $\cap$. In particular, if moreover $\{J \mid \delta(\Theta, J)=0\} \neq \emptyset$ (including the case where $\{J \mid \delta(\Theta, J)=0\}=\{\emptyset\}$ ), it has a (unique) maximal element $J_{0}$. 
Proof. Let $I, J \subset\{1,2, \ldots, k-1\}$. Then we can easily prove that

$$
\begin{aligned}
& \operatorname{dim}\left(\gamma_{k}^{\Theta}+\sum_{j \in I} \kappa_{j}^{\Theta}\right)+\operatorname{dim}\left(\gamma_{k}^{\Theta}+\sum_{j \in J} \kappa_{j}^{\Theta}\right) \\
& \geq \operatorname{dim}\left(\gamma_{k}^{\Theta}+\sum_{j \in I \cap J} \kappa_{j}^{\Theta}\right)+\operatorname{dim}\left(\gamma_{k}^{\Theta}+\sum_{j \in I \cup J} \kappa_{j}^{\Theta}\right) .
\end{aligned}
$$

Combining this inequality with the one $\sharp I+\sharp J=\sharp(I \cap J)+\sharp(I \cup J)$, we obtain

$$
\delta(\Theta, I)+\delta(\Theta, J) \geq \delta(\Theta, I \cap J)+\delta(\Theta, I \cup J) \geq 0,
$$

from which the assertion immediately follows.

Definition 5.3. We define an integer $E(\Theta)_{\lambda}$ to be 0 if $\min _{J \subset\{1,2, \ldots, k-1\}} \delta(\Theta, J) \neq 0$, and otherwise by using the maximal element $J_{0}$ of $\{J \mid \delta(\Theta, J)=0\}$ we set

$$
E(\Theta)_{\lambda}= \begin{cases}\operatorname{MV}\left(\kappa_{j_{1}}^{\Theta}, \kappa_{j_{2}}^{\Theta}, \ldots, \kappa_{j_{m}}^{\Theta}\right) & \left(\lambda^{d_{\Theta, J_{0}}}=1\right), \\ 0 & \text { (otherwise) }\end{cases}
$$

where $J_{0}=\left\{j_{1}, j_{2}, \ldots, j_{m}\right\}, \quad \sharp J_{0}=m$ and $\operatorname{MV}\left(\kappa_{j_{1}}^{\Theta}, \ldots, \kappa_{j_{m}}^{\Theta}\right) \in \mathbb{Z}_{+}$is the normalized $m$-dimensional mixed volume of $\kappa_{j_{1}}^{\Theta}, \ldots, \kappa_{j_{m}}^{\Theta}$. Note that by $\delta\left(\Theta, J_{0}\right)=0$ we have $\operatorname{dim}\left(\kappa_{j_{1}}^{\Theta}+\cdots+\kappa_{j_{m}}^{\Theta}\right) \leq \sharp J_{0}=m$ and the $m$-dimensional mixed volume $\operatorname{MV}\left(\kappa_{j_{1}}^{\Theta}, \ldots, \kappa_{j_{m}}^{\Theta}\right)$ makes sense.

In particular, if $\min _{J \subset\{1,2, \ldots, k-1\}} \delta(\Theta, J)=0$ and $\{J \mid \delta(\Theta, J)=0\}=\{\emptyset\}$ we set $E(\Theta)_{\lambda}=1$ or 0 depending on whether $\lambda^{d\left(\gamma_{k}^{\Theta}\right)}=1$ or not.

\section{Theorem 5.4 .}

(1) The degree of the virtual Betti polynomial $\beta\left(\mathcal{S}_{g, 0}\right)_{\lambda} \in \mathbb{Z}[t](\lambda \neq 1)$ is bounded by $2 n-2 k$. In particular, the sizes of the Jordan blocks for the eigenvalues $\lambda \neq 1$ in $\Phi_{n-k, 0}$ are bounded by $n-k+1$. 
(2) The number of the Jordan blocks for the eigenvalue $\lambda \neq 1$ with the maximal possible size $n-k+1$ in $\Phi_{n-k, 0}$ is equal to

$$
\sum_{\substack{\Theta \subset \Gamma_{f}, s_{\Theta}=n, \operatorname{dim} \Theta \geq k-1}}(-1)^{\operatorname{dim} \Theta-(k-1)} E(\Theta)_{\lambda} .
$$

Proof. (A) Assume that there exists $J \subset\{1,2, \ldots, k-1\}$ such that $\delta(\Theta, J)<0$. Then we have

$$
\delta(\Theta, J)=\operatorname{dim}\left(\Delta_{\gamma_{k}^{\Theta}}+\sum_{j \in J} \kappa_{j}^{\Theta}\right)-(\sharp J+1)<0 .
$$

By the dimensional reason, as a nondegenerate C.I. in $\tilde{T}_{\Theta}$ we have

$$
\left\{g_{j}^{\Theta}(x)=0(j \in J), \tilde{g}_{k}^{\Theta}(x)=0\right\}=\emptyset .
$$

Since $Z_{\Delta_{\Theta}}^{*} \subset \tilde{T}_{\Theta}$ is contained in this set, we obtain $Z_{\Delta_{\Theta}}^{*}=\emptyset$ and hence

$$
\frac{(-1)^{m_{\Theta}}}{t^{2 k-2}} \sum_{J \subset\{1,2, \ldots, k-1\}} \beta\left(\Theta_{J}, s_{\Theta}+\sharp J-1\right)_{\lambda}=0 .
$$

(B) Next assume that $\delta(\Theta, J) \geq 0$ for any $J \subset\{1,2, \ldots, k-1\}$. Then for any $J \subset\{1,2, \ldots, k-1\}$ we have

$$
\begin{aligned}
& \operatorname{deg}\left\{\frac{(-1)^{m_{\Theta}}}{t^{2 k-2}} \beta\left(\Theta_{J}, s_{\Theta}+\sharp J-1\right)_{\lambda}\right\} \\
& \leq 2\left(s_{\Theta}+\sharp J-1-\operatorname{dim} \Theta_{J}\right)+\operatorname{dim} \Theta_{J}-2 k+2 \\
& =2 s_{\Theta}-2 k+\sharp J-\operatorname{dim}\left(\gamma_{k}^{\Theta}+\sum_{j \in J} \kappa_{j}^{\Theta}\right) \\
& \leq 2 s_{\Theta}-2 k \leq 2 n-2 k .
\end{aligned}
$$

So the assertion (1) was proved. By the above calculations, if $s_{\Theta}<n$ or $\delta(\Theta, J)>0$ for any $J \subset\{1,2, \ldots, k-1\}$ there is no contribution to the leading coefficient $\beta_{2 n-2 k}\left(\mathcal{S}_{g, 0}\right)_{\lambda}$ from the face $\Theta \prec \Gamma_{+}(f)$. Therefore, to prove the assertion (2), we have only to consider 
the compact faces $\Theta \prec \Gamma_{+}(f)$ such that $s_{\Theta}=n$ and $\min _{J \subset\{1,2, \ldots, k-1\}} \delta(\Theta, J)=0$. In this case, for the maximal element $J_{0}$ of the set $\{J \mid \delta(\Theta, J)=0\}$ we have

$$
J \not \subset J_{0} \Longrightarrow \operatorname{deg}\left\{\frac{(-1)^{m_{\Theta}}}{t^{2 k-2}} \beta\left(\Theta_{J}, s_{\Theta}+\sharp J-1\right)_{\lambda}\right\}<2 n-2 k .
$$

This implies that

$$
\sum_{J \subset\{1,2, \ldots, k-1\}} \frac{(-1)^{m_{\Theta}}}{t^{2 k-2}} \beta\left(\Theta_{J}, s_{\Theta}+\sharp J-1\right)_{\lambda} \equiv \sum_{J \subset J_{0}} \frac{(-1)^{m_{\Theta}}}{t^{2 k-2}} \beta\left(\Theta_{J}, s_{\Theta}+\sharp J-1\right)_{\lambda}
$$

modulo polynomials of degree less than $2 n-2 k$. Set $J_{0}=\left\{j_{1}, j_{2}, \ldots, j_{m}\right\}, \sharp J_{0}=m$ and let

$$
Z_{\Delta_{\Theta}^{0}}^{*}=\left\{g_{j_{1}}^{\Theta}(x)=\cdots=g_{j_{m}}^{\Theta}(x)=\tilde{g}_{k}^{\Theta}(x)=0\right\} \subset\left(\mathbb{C}^{*}\right)^{S_{\Theta}}=\left(\mathbb{C}^{*}\right)^{n}
$$

be the nondegenerate complete intersection subvariety of codimension $m+1$ in $\left(\mathbb{C}^{*}\right)^{n}$ with a natural action of $\mu_{d_{\Theta}}$. Then by the arguments in Section 4 we have

$$
\sum_{J \subset J_{0}} \frac{1}{t^{2 m}} \beta\left(\Theta_{J}, n+\sharp J-1\right)_{\lambda}=\beta\left(Z_{\Delta_{\Theta}^{0}}^{*}\right)_{\lambda}
$$

Moreover, since by $\delta\left(\Theta, J_{0}\right)=0$ we have $\operatorname{dim}\left(\Delta_{\gamma_{k}^{\Theta}}+\sum_{j \in J_{0}} \kappa_{j}^{\Theta}\right)=\sharp J_{0}+1=m+1$, we can take another 0 -dimensional nondegenerate complete intersection subvariety

$$
D_{\Delta_{\Theta}^{0}}^{*}=\left\{g_{j_{1}}^{\Theta}(x)=\cdots=g_{j_{m}}^{\Theta}(x)=\tilde{g}_{k}^{\Theta}(x)=0\right\} \subset\left(\mathbb{C}^{*}\right)^{m+1}
$$

in $\left(\mathbb{C}^{*}\right)^{m+1}$ such that $Z_{\Delta_{\Theta}^{*}}^{*} \simeq\left(\mathbb{C}^{*}\right)^{n-m-1} \times D_{\Delta_{\Theta}^{*}}^{*}$. This implies that the right hand side of (115) is equal to

$$
\frac{(-1)^{m_{\Theta}}}{t^{2 k-2 m-2}}\left(t^{2}-1\right)^{n-m-1} \beta\left(D_{\Delta_{\Theta}^{0}}^{*}\right) \lambda
$$

whose leading coefficient (of degree $2 n-2 k$ ) is $(-1)^{m_{\Theta}} \beta\left(D_{\Delta_{\Theta}^{0}}^{*}\right)_{\lambda}=(-1)^{n-\operatorname{dim} \Theta-1} \beta_{0}\left(D_{\Delta_{\Theta}^{0}}^{*}\right)_{\lambda} \in$ $\mathbb{Z}$. By Theorem 2.6, the subset

$$
\left\{g_{j_{1}}^{\Theta}(x)=\cdots=g_{j_{m}}^{\Theta}(x)=0\right\} \subset\left(\mathbb{C}^{*}\right)^{m+1}
$$

of $\left(\mathbb{C}^{*}\right)^{m+1}$ is a disjoint union of $\operatorname{MV}\left(\kappa_{j_{1}}^{\Theta}, \ldots, \kappa_{j_{m}}^{\Theta}\right)$ copies of the complex line $\mathbb{C}$ and the restriction of $\tilde{g}_{k}^{\Theta}$ to each line vanishes at exactly $d_{\Theta, J_{0}}$ distinct points. Moreover we can easily see that the action of the generator of $\mu_{d_{\Theta}}$ on these $d_{\Theta, J_{0}}$ points corresponds to 
that of their automorphism group $\mu_{d_{\vartheta, J_{0}}}$. Then the assertion (2) follows. This completes the proof.

Remark 5.5. The maximal Jordan blocks in monodromies are important also in the monodromy conjecture. For the case $k=1$, see the recent result in Melle Hernández et al. [30].

As we see in the following corollary, our result becomes very simple when the dual fans of $\Gamma_{+}\left(f_{1}\right), \ldots, \Gamma_{+}\left(f_{k}\right)$ in $\mathbb{R}_{+}^{n}$ are the same. For example, if $\Gamma_{+}\left(f_{1}\right), \ldots, \Gamma_{+}\left(f_{k}\right)$ are similar, this condition is satisfied.

\section{Corollary 5.6.}

(1) If $k=2$, then the number of the Jordan blocks for the eigenvalue $\lambda \neq 1$ with the maximal possible size $n-k+1=n-1$ in $\Phi_{n-k, 0}$ is equal to

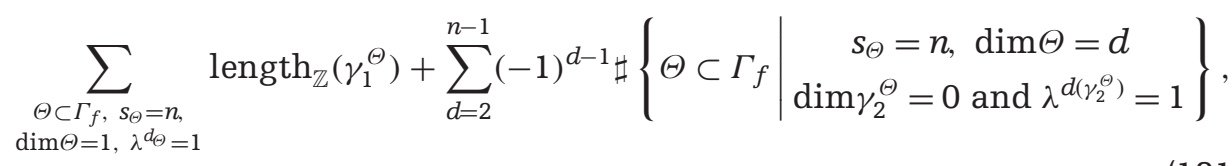

where length $\mathbb{Z}_{\mathbb{Z}}\left(\gamma_{1}^{\Theta}\right) \in \mathbb{Z}_{+}$is the lattice length of the segment $\gamma_{1}^{\Theta}$.

(2) Assume that the dual fans of $\Gamma_{+}\left(f_{1}\right), \ldots, \Gamma_{+}\left(f_{k}\right)$ in $\mathbb{R}_{+}^{n}$ are the same. Then the number of the Jordan blocks for the eigenvalue $\lambda \neq 1$ with the maximal possible size $n-k+1$ in $\Phi_{n-k, 0}$ is equal to

$$
\sum_{\substack{\Theta \subset \Gamma_{f}, s_{\Theta}=n, \operatorname{dim} \Theta=k-1, \lambda^{d}=1}} \operatorname{MV}\left(\kappa_{1}^{\Theta}, \ldots, \kappa_{k-1}^{\Theta}\right)
$$

where $\operatorname{MV}\left(\kappa_{1}^{\Theta}, \ldots, \kappa_{k-1}^{\Theta}\right) \in \mathbb{Z}_{+}$is the normalized $(k-1)$-dimensional mixed volume of $\kappa_{1}^{\Theta}, \ldots, \kappa_{k-1}^{\Theta}$.

Example 5.7. Assume that $n=3$ and $k=2$. Let

$$
W=\left\{f_{1}=0\right\} \supset V=\left\{f_{1}=f_{2}=0\right\}
$$

be complete intersection subvarieties of $\mathbb{C}^{3}$ having isolated singular points at the origin $0 \in \mathbb{C}^{3}$. Denote by $F_{0}$ the Milnor fiber of $g:=\left.f_{2}\right|_{W}: W \longrightarrow \mathbb{C}$ at the origin 0 . Assume also 
that the vertices of $\Gamma_{+}\left(f_{1}\right)$ (resp. $\left.\Gamma_{+}\left(f_{2}\right)\right)$ are $(3,0,0),(0,3,0)$ and $(0,0,3)$ (resp. $(2,2,2)$, $(8,0,0),(0,8,0)$ and $(0,0,8))$ and $f=\left(f_{1}, f_{2}\right)$ is nondegenerate at the origin $0 \in \mathbb{C}^{3}$. Then by the theorem of Oka [33,34] and Kirillov [20], the total multiplicity of the eigenvalue -1 in the monodromy operator $\Phi_{1,0}: H^{1}\left(F_{0} ; \mathbb{C}\right) \simeq H^{1}\left(F_{0} ; \mathbb{C}\right)$ is 18 . By Corollary 5.6(1) the number of the Jordan blocks for the eigenvalue -1 with the maximal possible size $n-k+1=2$ in $\Phi_{1,0}$ is $3 \times 3-1=8$. Hence the number of the Jordan blocks for the eigenvalue -1 with the size 1 in $\Phi_{1,0}$ is $18-2 \times 8=2$. We can check this result also by Corollary 5.15(1).

From now on, we shall describe the numbers of the second maximal Jordan blocks for the eigenvalues $\lambda \neq 1$ in the monodromy $\Phi_{n-k, 0}: H^{n-k}\left(F_{0} ; \mathbb{C}\right) \simeq H^{n-k}\left(F_{0} ; \mathbb{C}\right)$. We fix $\lambda \in \mathbb{C}^{*} \backslash\{1\}$ and a face $\Theta \prec \Gamma_{+}(f)$ such that $\Theta \subset \Gamma_{f}$. Recall that for any $J \subset$ $\{1,2, \ldots, k-1\}$ the Minkowski sum $\gamma_{k}^{\Theta}+\sum_{j \in J} \kappa_{j}^{\Theta}$ majorizes $\kappa_{j}^{\Theta}(j \in J)$ and $\gamma_{k}^{\Theta}$. For a face $\Gamma$ of $\gamma_{k}^{\Theta}+\sum_{j \in J} \kappa_{j}^{\Theta}$ denote by $\Gamma_{j}^{\Theta}(j \in J \sqcup\{k\})$ the corresponding faces of $\kappa_{j}^{\Theta}(j \in J)$ and $\gamma_{k}^{\Theta}$. Moreover for such $J$ and $\Gamma$ let $K_{\Theta, J}^{\Gamma}$ be the affine linear subspace of $\tilde{\mathbb{L}}_{\Theta} \simeq \mathbb{R}^{\operatorname{dim} \Theta+1}$ which is parallel to the affine span of $\Gamma$ and contains $\Gamma_{k}^{\Theta}$. Then we define an integer $d_{\Theta, J}^{\Gamma}>0$ to be the lattice distance of $K_{\Theta, J}^{\Gamma}$ from the origin $0 \in \tilde{\mathbb{L}}_{\Theta}$.

\section{Lemma 5.8.}

(1) Assume that $\min _{J \subset\{1,2, \ldots, k-1\}} \delta(\Theta, J)=1$. Then the set $\{J \mid \delta(\Theta, J)=1\} \neq \emptyset$ has a unique maximal element.

(2) Assume that $\min _{J \subset\{1,2, \ldots, k-1\}} \delta(\Theta, J)=0$ and let $J_{0}$ be the (unique) maximal element of $\{J \mid \delta(\Theta, J)=0\}$. Assume also that the set $\left\{J \mid \delta(\Theta, J)=1, J_{0} \subset J\right\}$ is not empty and let $I$ and $J$ be its maximal elements. Then we have $I=J$ or $I \cap J=J_{0}$.

Proof. (1) Let $I$ and $J$ be maximal elements of $\{J \mid \delta(\Theta, J)=1\} \neq \emptyset$. Then by the proof of Lemma 5.2 we have

$$
2=\delta(\Theta, I)+\delta(\Theta, J) \geq \delta(\Theta, I \cap J)+\delta(\Theta, I \cup J) \geq 2 .
$$

Since $\delta(\Theta, I \cap J), \delta(\Theta, I \cup J) \geq 1$ we obtain $\delta(\Theta, I \cup J)=1$. Then by the maximality of $I$ and $J$ we have $I=J$. 
(2) Assume that $I \neq J$. Then by the proof of Lemma 5.2 we have

$$
2=\delta(\Theta, I)+\delta(\Theta, J) \geq \delta(\Theta, I \cap J)+\delta(\Theta, I \cup J) \geq 0 .
$$

Since $\delta(\Theta, I \cup J) \geq 2$ by the maximality of $I$ and $J$, we obtain $\delta(\Theta, I \cap J)=0$ and hence $I \cap J=J_{0}$.

\section{Definition 5.9.}

(1) Assume that $\min _{J \subset\{1,2, \ldots, k-1\}} \delta(\Theta, J)=1$. Then we denote by $J_{1}$ the (unique) maximal element of $\{J \mid \delta(\Theta, J)=1\} \neq \emptyset$.

(2) Assume that $\min _{J \subset\{1,2, \ldots, k-1\}} \delta(\Theta, J)=0$. Then we define $n_{\Theta} \geq 0$ to be the number of the maximal elements of the set $\left\{J \mid \delta(\Theta, J)=1, J_{0} \subset J\right\}$. If $n_{\Theta}>0$ we denote by $J_{1}, J_{2}, \ldots, J_{n_{\Theta}}$ the maximal elements.

Lemma 5.10. Assume that $\min _{J \subset\{1,2, \ldots, k-1\}} \delta(\Theta, J)=0$. Then for any $J \subset\{1,2, \ldots, k-1\}$ such that $\delta(\Theta, J)=1$ we have $J \subset J_{0}$ or $\left(n_{\Theta}>0\right.$ and) $J \subset J_{i}$ for a unique $1 \leq i \leq n_{\Theta}$.

Proof. Assume that $\delta(\Theta, J)=1$ and $J \not \subset J_{0}$. Then by the proof of Lemma 5.2 we have

$$
1=\delta\left(\Theta, J_{0}\right)+\delta(\Theta, J) \geq \delta\left(\Theta, J_{0} \cap J\right)+\delta\left(\Theta, J_{0} \cup J\right) \geq 0 .
$$

Since $\delta\left(\Theta, J_{0} \cup J\right)=1$ by the maximality of $J_{0}$, we see that $n_{\Theta}>0$ and $J \subset J_{0} \cup J \subset J_{i}$ for some $1 \leq i \leq n_{\Theta}$. The uniqueness of $J_{i}$ such that $J \subset J_{i}$ follows from Lemma 5.8(2).

\section{Definition 5.11.}

(1) For $J \subset\{1,2, \ldots, k-1\}$ such that $\delta(\Theta, J)=0$, we set

$$
\operatorname{MV}\left(\kappa_{j}^{\Theta}(j \in J)\right)_{\lambda}= \begin{cases}\operatorname{MV}\left(\kappa_{j_{1}}^{\Theta}, \ldots, \kappa_{j_{m}}^{\Theta}\right) & \left(\lambda^{d_{\theta, J}}=1\right), \\ 0 & (\text { otherwise }),\end{cases}
$$

where $J=\left\{j_{1}, j_{2}, \ldots, j_{m}\right\}, \sharp J=m$ and $\operatorname{MV}\left(\kappa_{j_{1}}^{\Theta}, \ldots, \kappa_{j_{m}}^{\Theta}\right) \in \mathbb{Z}_{+}$is the normalized $m$-dimensional mixed volume. 
(2) For $J \subset\{1,2, \ldots, k-1\}$ such that $\delta(\Theta, J)=1$ we set

$$
\operatorname{MV}\left(\kappa_{j}^{\Theta}(j \in J), \gamma_{k}^{\Theta}+\sum_{j \in J} \kappa_{j}^{\Theta}\right)_{\lambda}= \begin{cases}\operatorname{MV}\left(\kappa_{j_{1}}^{\Theta}, \ldots, \kappa_{j_{m}}^{\Theta}, \gamma_{k}^{\Theta}+\sum_{i=1}^{m} \kappa_{j_{i}}^{\Theta}\right) & \left(\lambda^{d_{\Theta, J}}=1\right), \\ 0 & \text { (otherwise) }\end{cases}
$$

where $J=\left\{j_{1}, j_{2}, \ldots, j_{m}\right\}, \sharp J=m$ and $\operatorname{MV}\left(\kappa_{j_{1}}^{\Theta}, \ldots, \kappa_{j_{m}}^{\Theta}, \gamma_{k}^{\Theta}+\sum_{i=1}^{m} \kappa_{j_{i}}^{\Theta}\right) \in \mathbb{Z}_{+}$is the normalized $(m+1)$-dimensional mixed volume.

(3) For $J \subset\{1,2, \ldots, k-1\}$ such that $\delta(\Theta, J)=1$ and a facet $\Gamma$ of the $(\sharp J+1)$ dimensional Minkowski sum $\gamma_{k}^{\Theta}+\sum_{j \in J} \kappa_{j}^{\Theta}$ we set

$$
\operatorname{MV}\left(\Gamma_{j}^{\Theta}(j \in J)\right)_{\lambda}= \begin{cases}\operatorname{MV}\left(\Gamma_{j_{1}}^{\Theta}, \ldots, \Gamma_{j_{m}}^{\Theta}\right) & \left(\lambda^{\left.d_{\Theta, J}^{\Gamma}=1\right)}\right. \\ 0 & \text { (otherwise) }\end{cases}
$$

where $J=\left\{j_{1}, j_{2}, \ldots, j_{m}\right\}, \sharp J=m$ and $\operatorname{MV}\left(\Gamma_{j_{1}}^{\Theta}, \ldots, \Gamma_{j_{m}}^{\Theta}\right) \in \mathbb{Z}_{+}$is the normalized $m$-dimensional mixed volume.

Definition 5.12. For $\lambda \in \mathbb{C}^{*} \backslash\{1\}$ and a face $\Theta \prec \Gamma_{+}(f)$ such that $\Theta \subset \Gamma_{f}$ we define an integer $F(\Theta)_{\lambda} \in \mathbb{Z}$ as follows.

(1) If $\min _{J \subset\{1,2, \ldots, k-1\}} \delta(\Theta, J)<0$ or $>1$ we set $F(\Theta)_{\lambda}=0$.

(2) If $\min _{J \subset\{1,2, \ldots, k-1\}} \delta(\Theta, J)=1$, then by using the maximal element $J_{1}$ of $\{J \mid \delta(\Theta, J)=1\}$ we set

$$
F(\Theta)_{\lambda}=\operatorname{MV}\left(\kappa_{j}^{\Theta}\left(j \in J_{1}\right), \gamma_{k}^{\Theta}+\sum_{j \in J_{1}} \kappa_{j}^{\Theta}\right)_{\lambda}-\sum_{\Gamma} \operatorname{MV}\left(\Gamma_{j}^{\Theta}\left(j \in J_{1}\right)\right)_{\lambda}
$$

where in the sum $\sum_{\Gamma}$ the face $\Gamma$ ranges through the facets of $\gamma_{k}^{\Theta}+\sum_{j \in J_{1}} \kappa_{j}^{\Theta}$.

(3) If $\min _{J \subset\{1,2, \ldots, k-1\}} \delta(\Theta, J)=0$ and $n_{\Theta}=0 \Leftrightarrow\left\{J \mid \delta(\Theta, J)=1, J \not \subset J_{0}\right\}=\emptyset$ by Lemma 5.10), then we set $F(\Theta)_{\lambda}=0$. 
(4) If $\min _{J \subset\{1,2, \ldots, k-1\}} \delta(\Theta, J)=0$ and $n_{\Theta}>0$ we set

$$
\begin{aligned}
F(\Theta)_{\lambda}= & \sum_{i=1}^{n_{\Theta}}\left\{2 \operatorname{MV}\left(\kappa_{j}^{\Theta}\left(j \in J_{0}\right)\right)_{\lambda}+\operatorname{MV}\left(\kappa_{j}^{\Theta}\left(j \in J_{i}\right), \gamma_{k}^{\Theta}+\sum_{j \in J_{i}} \kappa_{j}^{\Theta}\right)_{\lambda}\right. \\
& \left.-\sum_{\Gamma} \operatorname{MV}\left(\Gamma_{j}^{\Theta}\left(j \in J_{i}\right)\right)_{\lambda}\right\},
\end{aligned}
$$

where in the sum $\sum_{\Gamma}$ the face $\Gamma$ ranges through the facets of $\gamma_{k}^{\Theta}+\sum_{j \in J_{i}} \kappa_{j}^{\Theta} \square$

Theorem 5.13. The number of the Jordan blocks for the eigenvalue $\lambda \neq 1$ with the second maximal possible size $n-k$ in $\Phi_{n-k, 0}$ is equal to

$$
\sum_{\substack{\Theta \subset \Gamma_{f}, s_{\Theta}=n, \operatorname{dim} \Theta \geq k}}(-1)^{\operatorname{dim} \Theta-k} F(\Theta)_{\lambda}
$$

Proof. It suffices to calculate the coefficient of $t^{2 n-2 k-1}$ of

$$
\left(1-t^{2}\right)^{m_{\Theta}} \beta\left(Z_{\Delta_{\Theta}}^{*}\right)_{\lambda}=\frac{(-1)^{m_{\Theta}}}{t^{2 k-2}} \sum_{J \subset\{1,2, \ldots, k-1\}} \beta\left(\Theta_{J}, s_{\Theta}+\sharp J-1\right)_{\lambda}
$$

for each face $\Theta \prec \Gamma_{+}(f)$ such that $\Theta \subset \Gamma_{f}$. By the proof of Theorem 5.4, this coefficient is zero unless $s_{\Theta}=n$ and there exists $J \subset\{1,2, \ldots, k-1\}$ such that $\delta(\Theta, J)=0,1$. Here we calculate only the contribution to the coefficient of $t^{2 n-2 k-1}$ from $\Theta$ such that $\Theta \subset$ $\Gamma_{f}, s_{\Theta}=n, \min _{J \subset\{1,2, \ldots, k-1\}} \delta(\Theta, J)=0$ and $n_{\Theta}>0$ (other cases can be treated similarly by using the proof of Theorem 5.4). In this case, by Lemma 5.10 we have

$$
\begin{aligned}
& \frac{(-1)^{m_{\Theta}}}{t^{2 k-2}} \sum_{J \subset\{1,2, \ldots, k-1\}} \beta\left(\Theta_{J}, s_{\Theta}+\sharp J-1\right)_{\lambda} \\
& \equiv \frac{(-1)^{m_{\Theta}}}{t^{2 k-2}}\left[\sum_{i=1}^{n_{\Theta}}\left\{\sum_{J \subset J_{i}} \beta\left(\Theta_{J}, n+\sharp J-1\right)_{\lambda}\right\}-\left(n_{\Theta}-1\right) \sum_{J \subset J_{0}} \beta\left(\Theta_{J}, n+\sharp J-1\right)_{\lambda}\right]
\end{aligned}
$$

modulo polynomials of degree less than $2 n-2 k-1$. By the proof of Theorem 5.4 the contribution to the coefficient of $t^{2 n-2 k-1}$ from the second term is zero. Moreover for 
each $1 \leq i \leq n_{\Theta}$ the coefficient of $t^{2 n-2 k-1}$ of

$$
\frac{(-1)^{m_{\Theta}}}{t^{2 k-2}} \sum_{J \subset J_{i}} \beta\left(\Theta_{J}, n+\sharp J-1\right)_{\lambda}
$$

is calculated as follows. Set $J_{i}=\left\{j_{1}, j_{2}, \ldots, j_{m}\right\}, \sharp J_{i}=m$ and let

$$
Z_{\Delta_{\Theta}^{i}}^{*}=\left\{g_{j_{1}}^{\Theta}(x)=\cdots=g_{j_{m}}^{\Theta}(x)=\tilde{g}_{k}^{\Theta}(x)=0\right\} \subset\left(\mathbb{C}^{*}\right)^{s_{\Theta}}=\left(\mathbb{C}^{*}\right)^{n}
$$

be the nondegenerate complete intersection subvariety of codimension $m+1$ in $\left(\mathbb{C}^{*}\right)^{n}$ with a natural action of $\mu_{d_{\Theta}}$. Then by the proof of Theorem 5.4 we have

$$
\beta\left(Z_{\Delta_{\Theta}^{i}}^{*}\right)_{\lambda}=\frac{1}{t^{2 m}} \sum_{J \subset J_{i}} \beta\left(\Theta_{J}, n+\sharp J-1\right)_{\lambda} .
$$

Since by $\delta\left(\Theta, J_{i}\right)=1$ we have $\operatorname{dim}\left(\Delta_{\gamma_{k}^{\Theta}}+\sum_{j \in J_{i}} \kappa_{j}^{\Theta}\right)=\sharp J_{i}+1+1=m+2$, we can take a nondegenerate C.I. curve

$$
C_{\Delta_{\Theta}^{i}}^{*}=\left\{g_{j_{1}}^{\Theta}(x)=\cdots=g_{j_{m}}^{\Theta}(x)=\tilde{g}_{k}^{\Theta}(x)=0\right\} \subset\left(\mathbb{C}^{*}\right)^{m+2}
$$

in $\left(\mathbb{C}^{*}\right)^{m+2}$ such that $Z_{\Delta_{\Theta}^{i}}^{*} \simeq\left(\mathbb{C}^{*}\right)^{n-m-2} \times C_{\Delta_{\Theta}^{i}}^{*}$. Hence we obtain

$$
\frac{(-1)^{m_{\Theta}}}{t^{2 k-2}} \sum_{J \subset J_{i}} \beta\left(\Theta_{J}, n+\sharp J-1\right)_{\lambda}=\frac{(-1)^{n-\operatorname{dim} \Theta-1}}{t^{2 k-2 m-2}}\left(t^{2}-1\right)^{n-m-2} \beta\left(C_{\Delta_{\Theta}^{*}}^{*}\right)_{\lambda} .
$$

Since the coefficient of $t^{2 n-2 k-1}$ of the last term is $(-1)^{n-\operatorname{dim} \Theta-1} \beta_{1}\left(C_{\Delta_{\Theta}^{i}}^{*}\right)_{\lambda}$, the assertion follows from the following proposition.

Proposition 5.14. In the situation above, we have

$$
\beta_{1}\left(C_{\Delta_{\Theta}^{i}}^{*}\right)_{\lambda}=-2 \operatorname{MV}\left(\kappa_{j}^{\Theta}\left(j \in J_{0}\right)\right)_{\lambda}-\operatorname{MV}\left(\kappa_{j}^{\Theta}\left(j \in J_{i}\right), \gamma_{k}^{\Theta}+\sum_{j \in J_{i}} \kappa_{j}^{\Theta}\right)_{\lambda}+\sum_{\Gamma} \operatorname{MV}\left(\Gamma_{j}^{\Theta}\left(j \in J_{i}\right)\right)_{\lambda}
$$


Proof. By the Cayley trick we have

$$
\beta\left(C_{\Delta_{\Theta}^{i}}^{*}\right)_{\lambda}=\frac{1}{t^{2 m}} \sum_{J \subset J_{i}} \beta\left(Z_{J}^{*}\right)_{\lambda}
$$

where $Z_{J}^{*}$ is the nondegenerate hypersurface of $\left(\mathbb{C}^{*}\right)^{m+2+\sharp J}$ defined by

$$
Z_{J}^{*}=\left\{\left(x ; \alpha_{j}(j \in J)\right) \in\left(\mathbb{C}^{*}\right)^{m+2} \times\left(\mathbb{C}^{*}\right)^{\sharp J} \mid \sum_{j \in J} \alpha_{j} g_{j}^{\Theta}(x)+\tilde{g}_{k}^{\Theta}(x)=0\right\} \subset\left(\mathbb{C}^{*}\right)^{m+2+\sharp J}
$$

By a simple calculation, for any $J \subset J_{i}$ we see that $\operatorname{deg} \beta\left(Z_{J}^{*}\right)_{\lambda} \leq 2 m+2-\delta(\Theta, J) \leq 2 m+$ 2. Hence the leading coefficient of the polynomial $\beta\left(C_{\Delta_{\Theta}^{i}}^{*}\right)_{\lambda} \in \mathbb{Z}[t]$ is $\beta_{2}\left(C_{\Delta_{\Theta}^{i}}^{*}\right)_{\lambda}$ and equal to that of

$$
\frac{1}{t^{2 m}} \sum_{J \subset J_{0}} \beta\left(Z_{J}^{*}\right)_{\lambda}=\frac{1}{t^{2\left(m-\sharp J_{0}\right)}}\left(t^{2}-1\right)^{m-\sharp J_{0}+1} \beta\left(D_{\Delta_{\Theta}^{*}}^{*}\right)_{\lambda},
$$

where $D_{\Delta_{\Theta}^{0}}^{*} \subset\left(\mathbb{C}^{*}\right)^{\sharp J_{0}+1}$ is the 0 -dimensional nondegenerate C.I. in $\left(\mathbb{C}^{*}\right)^{\sharp J_{0}+1}$ in the proof of Theorem 5.4. Consequently we obtain

$$
\beta_{2}\left(C_{\Delta_{\Theta}^{*}}^{*}\right)_{\lambda}=\beta_{0}\left(D_{\Delta_{\Theta}^{*}}^{*}\right)_{\lambda}=\operatorname{MV}\left(\kappa_{j}^{\Theta}\left(j \in J_{0}\right)\right)_{\lambda}
$$

From now on, let us calculate the $\lambda$-Euler characteristic

$$
\chi\left(C_{\Delta_{\Theta}^{i}}^{*}\right)_{\lambda}=\beta_{0}\left(C_{\Delta_{\Theta}^{i}}^{*}\right)_{\lambda}+\beta_{1}\left(C_{\Delta_{\Theta}^{i}}^{*}\right)_{\lambda}+\beta_{2}\left(C_{\Delta_{\Theta}^{i}}^{*}\right)_{\lambda}
$$

of the C.I. curve $C_{\Delta_{\Theta}^{i}}^{*} \subset\left(\mathbb{C}^{*}\right)^{m+2}$. First, note that if $J$ satisfies the condition $\operatorname{dim}\left(\Delta_{\gamma_{k}^{\Theta}}+\right.$ $\left.\sum_{j \in J} \kappa_{j}^{\Theta}\right)<m+2$, we have $\chi\left(Z_{J}^{*}\right)_{\lambda}=\chi\left(Z_{J}^{*}\right)=0$. So in the sum

$$
\chi\left(C_{\Delta_{\Theta}^{i}}^{*}\right)_{\lambda}=\sum_{J \subset J_{i}} \chi\left(Z_{J}^{*}\right)_{\lambda}
$$

only the terms $\chi\left(Z_{J}^{*}\right)_{\lambda}$ for $J$ such that $\operatorname{dim}\left(\gamma_{k}^{\Theta}+\sum_{j \in J} \kappa_{j}^{\Theta}\right)=m+1$ can be nontrivial. By Theorem 3.6 this implies that we have

$$
\chi\left(C_{\Delta_{\Theta}^{i}}^{*}\right)_{\lambda}= \begin{cases}\frac{1}{d_{\Theta, J_{i}}} \chi\left(C_{\Delta_{\Theta}^{i}}^{*}\right) & \left(\lambda^{d_{\Theta, J_{i}}}=1\right), \\ 0 & \text { (otherwise) }\end{cases}
$$


Since $C_{\Delta_{\Theta}^{*}}^{*} \subset\left(\mathbb{C}^{*}\right)^{m+2}$ is a $d_{\Theta, J_{i}}$-fold covering of the C.I. curve

$$
\left\{g_{j_{1}}^{\Theta}(x)=\cdots=g_{j_{m}}^{\Theta}(x)=0\right\} \backslash\left\{g_{j_{1}}^{\Theta}(x)=\cdots=g_{j_{m}}^{\Theta}(x)=g_{k}^{\Theta}(x)=0\right\}
$$

in $\left(\mathbb{C}^{*}\right)^{m+1}$, its usual Euler characteristic $\chi\left(C_{\Delta_{\Theta}^{i}}^{*}\right)$ is calculated by Theorem 2.6 as

$$
\chi\left(C_{\Delta_{\Theta}^{i}}^{*}\right)=-d_{\Theta, J_{i}} \times \operatorname{MV}\left(\kappa_{j_{1}}^{\Theta}, \ldots, \kappa_{j_{m}}^{\Theta}, \gamma_{k}^{\Theta}+\sum_{i=1}^{m} \kappa_{j_{i}}^{\Theta}\right) .
$$

Hence we get

$$
\chi\left(C_{\Delta_{\Theta}^{i}}^{*}\right)_{\lambda}=-\operatorname{MV}\left(\kappa_{j}^{\Theta}\left(j \in J_{i}\right), \gamma_{k}^{\Theta}+\sum_{j \in J_{i}} \kappa_{j}^{\Theta}\right)_{\lambda} .
$$

Now denote by $\square$ the Minkowski sum $\Delta_{\gamma_{k}^{\Theta}}+\sum_{j \in J_{i}} \kappa_{j}^{\Theta}$ in $\mathbb{R}^{m+2}$. Then $\square$ is an $(m+2)$ dimensional polytope and majorizes $\kappa_{j}^{\Theta}\left(j \in J_{i}\right)$ and $\Delta_{\gamma_{k}^{\Theta}}$. Let $X_{\square}$ be the toric variety associated to the dual fan of $\square$. Then $X_{\square}$ is a compactification of the complex torus $T=\left(\mathbb{C}^{*}\right)^{m+2}$ and smooth outside the union of $T$-orbits of codimension $\geq 2$. Therefore, by the nondegeneracy of $C_{\Delta_{\Theta}^{*}}^{*} \subset\left(\mathbb{C}^{*}\right)^{m+2}$ its closure $\overline{C_{\Delta_{\Theta}^{*}}^{*}}$ in $X_{\square}$ is a smooth projective curve. By the Poincaré duality of $\overline{C_{\Delta_{\Theta}^{i}}^{*}}$ we have

$$
\beta_{0}\left(\overline{C_{\Delta_{\Theta}^{i}}^{*}}\right)_{\lambda}=\beta_{2}\left(\overline{C_{\Delta_{\Theta}^{i}}^{*}}\right)_{\lambda^{-1}}=\beta_{2}\left(C_{\Delta_{\Theta}^{i}}^{*}\right)_{\lambda^{-1}}=\operatorname{MV}\left(\kappa_{j}^{\Theta}\left(j \in J_{0}\right)\right)_{\lambda}
$$

and hence

$$
\beta_{0}\left(C_{\Delta_{\Theta}^{i}}^{*}\right)_{\lambda}=\operatorname{MV}\left(\kappa_{j}^{\Theta}\left(j \in J_{0}\right)\right)_{\lambda}-\sum_{\Gamma} \operatorname{MV}\left(\Gamma_{j}^{\Theta}\left(j \in J_{i}\right)\right)_{\lambda}
$$

Then we obtain the desired formula for the first virtual Betti number $\beta_{1}\left(C_{\Delta_{\Theta}^{*}}^{*}\right)_{\lambda}$. This completes the proof. 


\section{Corollary 5.15.}

(1) If $k=2$, then the number of the Jordan blocks for the eigenvalue $\lambda \neq 1$ with the second maximal possible size $n-k$ in $\Phi_{n-k, 0}$ is equal to

$$
\begin{aligned}
& \sum_{\substack{\Theta \subset \Gamma_{f}, s_{\Theta}=n, \operatorname{dim} \Theta=2}} F(\Theta)_{\lambda}+\sum_{d=3}^{n-1}(-1)^{d} \\
& \times\left\{\sum_{\substack{s_{\Theta}=n, \operatorname{dim} \Theta=d, \operatorname{dim} \gamma_{2}^{\Theta}=1, \lambda^{d\left(\gamma_{2}^{\Theta}\right)}=1}}\left(\text { length }_{\mathbb{Z}}\left(\gamma_{2}^{\Theta}\right)-\sharp\left\{v \prec \gamma_{2}^{\Theta} \mid \operatorname{dim} v=0, \lambda^{d(v)}=1\right\}\right)\right\} .
\end{aligned}
$$

(2) Assume that the dual fans of $\Gamma_{+}\left(f_{1}\right), \ldots, \Gamma_{+}\left(f_{k}\right)$ in $\mathbb{R}_{+}^{n}$ are the same. Then the number of the Jordan blocks for the eigenvalue $\lambda \neq 1$ with the second maximal possible size $n-k$ in $\Phi_{n-k, 0}$ is equal to

$$
\sum_{\substack{\Theta \subset \Gamma_{f}, s_{\Theta}=n, \operatorname{dim} \Theta=k}} F(\Theta)_{\lambda} .
$$

\section{Monodromies at Infinity over C.I.}

In this section, we study the monodromies at infinity over complete intersection subvarieties in $\mathbb{C}^{n}$. For $2 \leq k \leq n$ let

$$
W=\left\{f_{1}=\cdots=f_{k-1}=0\right\} \supset V=\left\{f_{1}=\cdots=f_{k-1}=f_{k}=0\right\}
$$

be complete intersection subvarieties of $\mathbb{C}^{n}$. Then for the polynomial map $f=$ $\left(f_{1}, f_{2}, \ldots, f_{k}\right): \mathbb{C}^{n} \longrightarrow \mathbb{C}^{k}$, there exists a complex hypersurface $D \subset \mathbb{C}^{k}$ such that the restriction $\mathbb{C}^{n} \backslash f^{-1}(D) \longrightarrow \mathbb{C}^{k} \backslash D$ of $f$ is a locally trivial fibration. We assume that the $k$ th coordinate axis

$$
A_{k}=\left\{y \in \mathbb{C}^{k} \mid y_{1}=y_{2}=\cdots=y_{k-1}=0\right\} \simeq \mathbb{C}
$$

satisfies the condition $\sharp\left(A_{k} \cap D\right)<+\infty$. Let $C_{R}$ be a circle in $A_{k} \simeq \mathbb{C}$ centered at the origin $0 \in A_{k}$ with a sufficiently large radius $R \gg 0$. Here we take $R$ large enough so that the open disk whose boundary is $C_{R}$ contains the finite set $A_{k} \cap D$. Let $g=\left.f\right|_{W}=\left.f_{k}\right|_{W}$ : $W=f^{-1}\left(A_{k}\right) \longrightarrow \mathbb{C} \simeq A_{k}$ be the restriction of $f$ to $W$. Then by restricting the locally 
trivial fibration $W \backslash g^{-1}\left(A_{k} \cap D\right) \longrightarrow A_{k} \backslash\left(A_{k} \cap D\right)$ to $C_{R} \subset \mathbb{C} \simeq A_{k}$, we obtain a geometric monodromy automorphism $\Phi^{\infty}: g^{-1}(R) \stackrel{\sim}{\longrightarrow} g^{-1}(R)$ and the linear maps

$$
\Phi_{j}^{\infty}: H^{j}\left(g^{-1}(R) ; \mathbb{C}\right) \stackrel{\sim}{\longrightarrow} H^{j}\left(g^{-1}(R) ; \mathbb{C}\right) \quad(j=0,1, \ldots)
$$

associated to it. We call $\Phi_{j}^{\infty}$ the (cohomological) $k$ th principal monodromies at infinity of $f$. For the case $k=1$ see $[2,14,28,29,36,41,42]$ etc. The semisimple parts of $\Phi_{j}^{\infty}$ were studied in [28, Section 5]. From now on, we shall determine their Jordan normal forms for the eigenvalues $\lambda \neq 1$. Note that if the generic fiber $g^{-1}(R)(R \gg 0)$ of $g$ satisfies the condition $H^{j}\left(g^{-1}(R) ; \mathbb{C}\right) \simeq 0(j \neq 0, n-k)$ (see, e.g., Tibăr [46, Theorem 6.2]) then it suffices to determine the Jordan normal form of $\Phi_{n-k}^{\infty}: H^{n-k}\left(g^{-1}(R) ; \mathbb{C}\right) \stackrel{\sim}{\longrightarrow} H^{n-k}\left(g^{-1}(R) ; \mathbb{C}\right)(R \gg 0)$. Let $j: \mathbb{C} \hookrightarrow \mathbb{P}^{1}$ be the inclusion and $h$ a local coordinate on a neighborhood of $\infty \in \mathbb{P}^{1}$ such that $\{\infty\}=h^{-1}(0)$. Then to the object $\psi_{h}\left(j_{!} R g_{!}\left(\mathbb{C}_{W}\right)\right) \in \mathbf{D}_{C}^{b}(\{\infty\})$ and the semisimple part of the monodromy automorphism acting on it, we associate naturally an element

$$
\left[H_{g}^{\infty}\right] \in \mathrm{K}_{0}\left(\mathrm{HS}^{\mathrm{mon}}\right) .
$$

Proposition 6.1. Assume that $W \subset \mathbb{C}^{n}$ has only isolated singular points, $g: W \longrightarrow \mathbb{C}$ is cohomologically tame in the sense of Sabbah [37] and the generic fiber $g^{-1}(R)(R \gg 0)$ of $g$ satisfies the condition $H^{j}\left(g^{-1}(R) ; \mathbb{C}\right) \simeq 0(j \neq 0, n-k)$. Let $\lambda \in \mathbb{C}^{*} \backslash\{1\}$. Then

(1) We have $e^{p, q}\left(\left[H_{g}^{\infty}\right]\right)_{\lambda}=0$ for $(p, q) \notin[0, n-k] \times[0, n-k]$. Moreover for $(p, q) \in$ $[0, n-k] \times[0, n-k]$ we have

$$
e^{p, q}\left(\left[H_{g}^{\infty}\right]\right)_{\lambda}=e^{n-k-q, n-k-p}\left(\left[H_{g}^{\infty}\right]\right)_{\lambda}
$$

(2) For $i \geq 1$, the number of the Jordan blocks for the eigenvalue $\lambda$ with sizes $\geq i$ in $\Phi_{n-k}^{\infty}: H^{n-k}\left(g^{-1}(R) ; \mathbb{C}\right) \stackrel{\sim}{\longrightarrow} H^{n-k}\left(g^{-1}(R) ; \mathbb{C}\right)(R \gg 0)$ is equal to

$$
(-1)^{n-k} \sum_{p+q=n-k-1+i, n-k+i} e^{p, q}\left(\left[H_{g}^{\infty}\right]\right)_{\lambda}
$$

Proof. Let $\mathcal{F} \in \mathbf{D}_{C}^{b}(W)$ be the intersection cohomology complex of $W$. Then by using a nice compactification of $g: W \longrightarrow \mathbb{C}$, we can prove an analogue of [37, Theorem 8.1(ii)] for the natural morphism $R g_{!} \mathcal{F} \longrightarrow R g_{*} \mathcal{F}$. Hence for $\lambda \neq 1$, the $\lambda$-part $\psi_{h, \lambda}\left(j ! R g_{!}\left(\mathbb{C}_{W}\right)\right) \in$ 
$\mathbf{D}_{c}^{b}(\{\infty\})$ is isomorphic to

$$
\psi_{h, \lambda}\left(j_{!} R g_{!}(\mathcal{F}[-n+k-1])\right) \simeq \psi_{h, \lambda}\left(R j_{*} R g_{*}(\mathcal{F}[-n+k-1])\right) .
$$

Then by the proof of Sabbah [37, Theorem 13.1], its relative monodromy filtration is the absolute one (up to a shift), and the assertions follow.

In order to rewrite this result explicitly, assume moreover that the polynomials $f_{1}, f_{2}, \ldots, f_{k}$ are convenient.

Definition $6.2([24,28]$ etc.).

(1) For $1 \leq j \leq k$ we call the convex hull of $\{0\} \cup \operatorname{NP}\left(f_{j}\right)$ in $\mathbb{R}^{n}$ the Newton polyhedron at infinity of $f_{j}$ and denote it by $\Gamma_{\infty}\left(f_{j}\right)$. Moreover we set

$$
\Gamma_{\infty}(f):=\Gamma_{\infty}\left(f_{1}\right)+\Gamma_{\infty}\left(f_{2}\right)+\cdots+\Gamma_{\infty}\left(f_{k}\right) .
$$

(2) We say that $\Theta \prec \Gamma_{\infty}(f)$ (resp. $\gamma \prec \Gamma_{\infty}\left(f_{j}\right)$ ) is a face at infinity if $0 \notin \Theta$ (resp. $0 \notin \gamma)$.

As in Section 4, for each face at infinity $\Theta$ of $\Gamma_{\infty}(f)$, we define those $\gamma_{j}^{\Theta}$ of $\Gamma_{\infty}\left(f_{j}\right)$ so that we have

$$
\Theta=\gamma_{1}^{\Theta}+\gamma_{2}^{\Theta}+\cdots+\gamma_{k}^{\Theta} .
$$

For $1 \leq j \leq k$ and a face at infinity $\Theta$ of $\Gamma_{\infty}(f)$, we define $f_{j}^{\Theta} \in \mathbb{C}\left[x_{1}, x_{2}, \ldots, x_{n}\right]$ as in Section 4 .

Definition 6.3 ([28]). We say that $f=\left(f_{1}, \ldots, f_{k}\right)$ is nondegenerate at infinity if for any face at infinity $\Theta$ of $\Gamma_{\infty}(f)$ the two subvarieties $\left\{f_{1}^{\Theta}(x)=\cdots=f_{k-1}^{\Theta}(x)=0\right\}$ and $\left\{f_{1}^{\Theta}(x)=\cdots=f_{k-1}^{\Theta}(x)=f_{k}^{\Theta}(x)=0\right\}$ in $\left(\mathbb{C}^{*}\right)^{n}$ are nondegenerate complete intersections.

From now on, let us assume also that $f$ is nondegenerate at infinity. Let $\Sigma_{1}$ be the dual fan of $\Gamma_{\infty}(f)$ and $\Sigma_{0}$ the fan formed by the faces of the first quadrant $\mathbb{R}_{+}^{n}$. By the convenience of $f_{1}, \ldots, f_{k}, \Sigma_{0}$ is a subfan of $\Sigma_{1}$ and hence we can construct a smooth subdivision $\Sigma$ of $\Sigma_{1}$ without subdividing the cones in $\Sigma_{0}$. Then the toric variety $X_{\Sigma}$ associated to $\Sigma$ is a smooth compactification of $\mathbb{C}^{n}$. By the nondegeneracy of $f$ at infinity, it follows from the construction of $X_{\Sigma}$ that $W \subset \mathbb{C}^{n}$ has only isolated singular points. 
By constructing a blow-up of $X_{\Sigma}$ (to eliminate the points of indeterminacy of the meromorphic extension of $f_{k}$ to $X_{\Sigma}$ ) as in [29], we can check also that $g: W \longrightarrow \mathbb{C}$ is cohomologically tame. Moreover by Tibăr [46, Theorem 6.2] the generic fiber $g^{-1}(R)(R \gg 0)$ of $g$ satisfies $H^{j}\left(g^{-1}(R) ; \mathbb{C}\right) \simeq 0(j \neq 0, n-k)$. Hence all the assumptions of Proposition 6.1 are satisfied. Now, as in [29, Section 4; 35] (see also [15]), by using the blow-up of $X_{\Sigma}$ we can construct an element $\mathcal{S}_{g}^{\infty} \in \mathcal{M}_{\mathbb{C}}^{\hat{\mu}}$ such that $\chi_{h}\left(\mathcal{S}_{g}^{\infty}\right)=\left[H_{g}^{\infty}\right]$. We call $\mathcal{S}_{g}^{\infty}$ the motivic Milnor fiber at infinity of $g: W \longrightarrow \mathbb{C}$. For each face at infinity $\Theta$ of $\Gamma_{\infty}(f)$ we define an element $\left[Z_{\Delta_{\Theta}}^{*}\right] \in \mathcal{M}_{\mathbb{C}}^{\hat{\mu}}$ and an integer $m_{\Theta} \in \mathbb{Z}_{+}$etc. as in Section 4 . Then the following result can be obtained in the same way as Theorem 4.7 (see the proof of [29, Theorems 5.3 and 7.3]).

Theorem 6.4. Assume that $\lambda \in \mathbb{C}^{*} \backslash\{1\}$. Then

(1) In the Grothendieck group $K_{0}(\mathrm{HS})$, we have

$$
\chi_{h}\left(\mathcal{S}_{g}^{\infty}\right)_{\lambda}=\sum_{0 \notin \Theta, \operatorname{dim} \Theta \geq k-1} \chi_{h}\left((1-\mathbb{L})^{m_{\Theta}} \cdot\left[Z_{\Delta_{\Theta}}^{*}\right]\right)_{\lambda} .
$$

In particular, we have

$$
\beta\left(\mathcal{S}_{g}^{\infty}\right)_{\lambda}=\sum_{0 \notin \Theta, \operatorname{dim} \Theta \geq k-1}\left(1-t^{2}\right)^{m_{\Theta}} \cdot \beta\left(Z_{\Delta_{\Theta}}^{*}\right)_{\lambda}
$$

(2) For $i \geq 1$, the number of the Jordan blocks for the eigenvalue $\lambda$ with sizes $\geq i$ in $\Phi_{n-k}^{\infty}: H^{n-k}\left(g^{-1}(R) ; \mathbb{C}\right) \stackrel{\sim}{\longrightarrow} H^{n-k}\left(g^{-1}(R) ; \mathbb{C}\right)(R \gg 0)$ is equal to

$$
(-1)^{n-k}\left\{\beta_{n-k-1+i}\left(\mathcal{S}_{g}^{\infty}\right)_{\lambda}+\beta_{n-k+i}\left(\mathcal{S}_{g}^{\infty}\right)_{\lambda}\right\}
$$

Moreover, also for $\beta\left(\mathcal{S}_{g}^{\infty}\right)_{\lambda} \in \mathbb{Z}[t](\lambda \neq 1)$ we can obtain a formula completely similar to Theorem 4.9. Therefore, we can always calculate the numbers of the Jordan blocks for the eigenvalues $\lambda \neq 1$ in $\Phi_{n-k}^{\infty}$ by the results in Sections 2 and 3. It is also clear that the analogues of the results in Sections 4 and 5 hold for $\Phi_{n-k}^{\infty}$. We thus find a striking symmetry between local and global. 


\section{Acknowledgements}

The authors express their hearty gratitude to Professors Y. Matsui, A. Melle Hernández, and C. Sabbah for their very fruitful discussions. They are also grateful to the referees who largely improved this paper.

\section{Funding}

Alexander Esterov is supported by Russian Foundation for Basic Research [RFBR-10-01-00678].

\section{References}

[1] A'Campo, N. "La fonction zêta d'une monodromie." Commentarii Mathematici Helvetici 50 (1975): 233-48.

[2] Broughton, S. A. "Milnor numbers and the topology of polynomial hypersurfaces." Inventiones Mathematicae 92, no. 2 (1988): 217-41.

[3] Danilov, V. I. "Newton polyhedra and vanishing cohomology." Functional Analysis and its Applications 13, no. 2 (1979): 103-15.

[4] Danilov, V. I. and A. G. Khovanskii. "Newton polyhedra and an algorithm for computing Hodge-Deligne numbers." Mathematics of the Ussr Izvestiya 29, no. 2 (1987): 279-98.

[5] Denef, J. and F. Loeser. "Motivic Igusa zeta functions." Journal of Algebraic Geometry 7 , no. 3 (1998): 505-37.

[6] Denef, J. and F. Loeser. "Geometry on arc spaces of algebraic varieties." Progress in Mathematics 201 (2001): 327-48.

[7] Dimca, A. "Monodromy and Betti numbers of weighted complete intersections." Topology 24, no. 3 (1985): 369-74.

[8] Dimca, A. Sheaves in Topology. Berlin: Universitext, Springer, 2004.

[9] Ebeling, W. and J. H. M. Steenbrink. "Spectral pairs for isolated compete intersection singularities." Journal of Algebraic Geometry 7, no. 1 (1998): 55-76.

[10] Esterov, A. "The index of a real singular point and its Newton diagram." Moscow University Mathematics Bulletin 58, no. 1 (2003): 7-11.

[11] Esterov, A. "Determinantal singularities and Newton polyhedra." Proceedings of the Steklov Institute of Mathematics 259, no. 1 (2007): 16-34, arXiv:0906.5097.

[12] Esterov, A. "Newton polyhedra of discriminants of projections." Discrete \& Computational Geometry 44, no. 1 (2010): 96-148, arXiv:0810.4996.

[13] Fulton, W. Introduction to Toric Varieties. Princeton, NJ: Princeton University Press, 1993.

[14] García López, R. and A. Némethi. "Hodge numbers attached to a polynomial map." Annales de l'Institut Fourier 49, no. 5 (1999): 1547-79.

[15] Guibert, G., F. Loeser, and M. Merle. "Iterated vanishing cycles, convolution, and a motivic analogue of a conjecture of Steenbrink." Duke Mathematical Journal 132, no. 3 (2006): 40957. 
[16] Hamm, H. "Lokale topologische Eigenschaften komplexer Räume." Mathematische Annalen 191 (1971): 235-52.

[17] Hotta, R., K. Takeuchi, and T. Tanisaki. D-modules, Perverse Sheaves and Representation Theory. Boston: Birkhäuser, 2008.

[18] Kashiwara, M. and P. Schapira. Sheaves on Manifolds. New York: Springer, 1990.

[19] Khovanskii, A. G. "Newton polyhedra and the genus of complete intersections." Functional Analysis and its Applications 12, no. 1 (1978): 38-46.

[20] Kirillov, A. N. "Zeta function of the monodromy for complete intersection singularities." Journal of Mathematical Sciences 25, no. 2 (1984): 1051-7.

[21] Kouchnirenko, A. G. "Polyèdres de Newton et nombres de Milnor." Inventiones Mathematicae 32, no. 1 (1976): 1-31.

[22] Kulikov, V. S. Mixed Hodge Structures and Singularities. Cambridge: Cambridge University Press, 1998.

[23] Lê, D. T. "Some Remarks on Relative Monodromy." Real and Complex Singularities (Proc. Ninth Nordic Summer School/NAVF Sympos. Math., Oslo, 1976) Sijthoff and Noordhoff, Alphen aan den Rijn (1977): 397-403.

[24] Libgober, A. and S. Sperber. "On the zeta function of monodromy of a polynomial map." Compositio Mathematica 95, no. 3 (1995): 287-307.

[25] Macdonald, I. G. "Polynomials associated with finite cell-complexes." Journal of the London Mathematical Society 4, no. 4 (1971): 181-92.

[26] Matsui, Y. and K. Takeuchi. "A geometric degree formula for A-discriminants and Euler obstructions of toric varieties." Advances in Mathematics 226, no. 2 (2011): 2040-64.

[27] Matsui, Y. and K. Takeuchi. "Milnor fibers over singular toric varieties and nearby cycle sheaves." Tohoku Mathematical Journal 63, no. 1 (2011): 113-36.

[28] Matsui, Y. and K. Takeuchi. "Monodromy zeta functions at infinity, Newton polyhedra and constructible sheaves." Mathematische Zeitschrift 268, no. 1-2 (2011): 409-39.

[29] Matsui, Y. and K. Takeuchi. "Monodromy at infinity of polynomial maps and mixed Hodge modules, with Appendix by C. Sabbah." (2009): submitted, arXiv:0912.5144v10.

[30] Melle Hernández, A., T. Torrelli, and W. Veys. “On 'maximal' poles of zeta functions, roots of b-functions and monodromy Jordan blocks." Journal of Topology 2, no. 3 (2009): 517-26.

[31] Milnor, J. Singular Points of Complex Hypersurfaces. Princeton, NJ: Princeton University Press, 1968.

[32] Oda, T. Convex Bodies and Algebraic Geometry. An Introduction to the Theory of Toric Varieties. New York: Springer, 1988.

[33] Oka, M. "Principal zeta-function of non-degenerate complete intersection singularity." Journal of Mathematical Sciences, The University of Tokyo 37, no. 1 (1990): 11-32.

[34] Oka, M. Non-degenerate Complete Intersection Singularity. Paris: Hermann, 1997.

[35] Raibaut, M. "Fibre de Milnor motivique à l'infini." Comptes Rendus de l'Académie des Sciences. Série I. Mathématique, 348, no. 7-8 (2010): 419-22.

[36] Sabbah, C. "Monodromy at infinity and Fourier transform." Publications of the Research Institute for Mathematical Sciences 33, no. 4 (1997): 643-85. 
[37] Sabbah, C. "Hypergeometric periods for a tame polynomial." Portugaliae Mathematica 63, no. 2 (2006): 173-226.

[38] Saito, M. "Modules de Hodge polarisables." Publications of the Research Institute for Mathematical Sciences 24, no. 6 (1988): 849-995.

[39] Saito, M. "Exponents and Newton polyhedra of isolated hypersurface singularities." Mathematische Annalen 281, no. 3 (1988): 411-7.

[40] Saito, M. "Mixed Hodge modules." Publications of the Research Institute for Mathematical Sciences 26, no. 2 (1990): 221-333.

[41] Siersma, D. and M. Tibăr. "Singularities at infinity and their vanishing cycles." Duke Mathematical Journal 80, no. 3 (1995): 771-83.

[42] Steenbrink, J. and S. Zucker. "Variation of mixed Hodge structure I." Inventiones Mathematicae 80, no. 3 (1985): 489-542.

[43] Sturmfels, B. "On the Newton polytope of the resultant." Journal of Algebraic Combinatorics 3, no. 2 (1994): 207-36.

[44] Takeuchi, K. "Perverse sheaves and Milnor fibers over singular varieties." Advanced Studies in Pure Mathematics 46 (2007): 211-222.

[45] Tanabé, S. "Combinatorial aspects of the mixed Hodge structure." RIMS Kôkyûroku 1374 (2004): 15-39.

[46] Tibăr, M. "Topology at infinity of polynomial mappings and Thom regularity condition." Compositio Mathematica 111, no. 1 (1998): 89-109.

[47] Varchenko, A. N. "Zeta-function of monodromy and Newton's diagram." Inventiones Mathematicae 37, no. 3 (1976): 253-62.

[48] Varchenko, A. N. and Khovanskii, A. G. "Asymptotic behavior of integrals over vanishing cycles and the Newton polyhedron." Dokl. Akad. Nauk SSSR 283 (1985): 521-5, Soviet Math. Doklady 32, no. 1 (1985): 122-7.

[49] Voisin, C. Hodge Theory and Complex Algebraic Geometry, I. Cambridge: Cambridge University Press, 2007. 